\title{
Synchronization of a Class of Chaotic Systems with Different Dimensions
}

\author{
Jiming Zheng $\mathbb{D}^{1}$ and Juan Li $\mathbb{D}^{2}$ \\ ${ }^{1}$ Key Laboratory of Intelligent Analysis and Decision Complex Systems, Chongqing University of Posts and Telecommunications, \\ Chongqing 400065, China \\ ${ }^{2}$ School of Science, Chongqing University of Posts and Telecommunications, Chongqing 400065, China
}

Correspondence should be addressed to Jiming Zheng; zhengjm0903@qq.com

Received 25 November 2020; Revised 22 January 2021; Accepted 29 March 2021; Published 14 April 2021

Academic Editor: Xianggui Guo

Copyright ( 92021 Jiming Zheng and Juan Li. This is an open access article distributed under the Creative Commons Attribution License, which permits unrestricted use, distribution, and reproduction in any medium, provided the original work is properly cited.

\begin{abstract}
In this paper, two scaling matrices are used to research the synchronization of different dimensional chaotic systems with unknown parameters. Firstly, the definition of synchronization of chaotic systems with different dimensions is introduced. Secondly, based on Lyapunov stability theorem and adaptive control method, an adaptive feedback hybrid controller and parameter adaptive laws are designed to realize synchronization of uncertain chaotic systems with different dimensions. Finally, three numerical experiments are carried out to verify the effectiveness of the proposed method.
\end{abstract}

\section{Introduction}

Chaos is a very complex nonlinear motion, which will show randomness, boundedness, unpredictability, and sensitive dependence on the initial value in the evolution process [1]. Chaotic system is different from the general system, and it is greatly affected by the changes in initial value and parameters, through the continuous in-depth understanding of the characteristics of chaotic motion, gradually realizing that the existence of chaos has brought some damage to the system; for example, chaotic motion can cause the circuit to form irregular oscillation and make the chaotic motion gradually away from the target trajectory, which may cause unpredictable consequences. At the same time, chaos also enlarges the influence of small changes and improves their effectiveness of achieving a specific desired state with very high flexibility and low cost. In some research fields, this property may be a valuable advantage. In 1990, Pecora and Carroll first realized chaos synchronization in electronic circuits [2], which set off an upsurge of chaos synchronization control research, making chaos synchronization widely used in many fields, such as communication encryption, engineering optimization, and statistical prediction [3-6].
In recent years, with the in-depth understanding of chaotic systems, some typical synchronization problems have been studied, such as complete synchronization, antisynchronization, projective synchronization, and hybrid synchronization [7-9]. However, in most of the control strategy design process, the system will be affected by various uncertain factors, and it is difficult to directly obtain some accurate mathematical models of the system. Some uncertain parameters of the chaotic system may have a certain impact on the performance of the system. On the contrary, due to the influence of these uncertain factors, the synchronization behavior of the system may be destroyed, and the emergence of unknown quantities in the system makes the synchronization control very complex. Many existing kinds of literatures consider the synchronization between two uncertain chaotic systems in the same dimension; for example, in [10], an adaptive sliding mode disturbance observer is designed to research the synchronization problem of uncertain Dadras-Momeni chaotic systems with time-varying disturbances. In [11], a finite-time synchronization scheme is proposed for uncertain chaotic systems with external disturbances, parametric uncertainties, Lipschitz nonlinearities, and time delays. In [12], an adaptive 
complete synchronization scheme is designed for uncertain financial chaotic systems with unknown parameters.

Synchronization behavior also exists in different dimensional systems in practice, and it is also especially important; for example, the circulatory and respiratory systems with different dimensions exist in synchronization behavior [13]. Similarly, in the communication network, the different dimension structure design and synchronization control of the driving system and the response system will increase the difficulty for the listener to decipher and make the communication more secure [14]. Therefore, it is of great significance to research the synchronization of chaotic systems with uncertain parameters and different dimensions in practical applications. In [15], the synchronization of a particular chaotic system with unknown parameters is studied by constructing the cross-error difference term. Recently, there have been some research studies on achieving the synchronization of chaotic systems by reducing dimension $[16,17]$, as well as some research studies by increasing dimension and reducing dimension simultaneously $[18,19]$. In $[20-22]$, the author further studies the synchronization of different dimensional integer-order or fractional-order chaotic or hyperchaotic systems using scaling matrices.

The main contribution of this paper is to research the scaling matrix synchronization of different dimensional chaotic or hyperchaotic systems with unknown parameters. A new suitable controller and parameter adaptive laws are designed to realize the synchronization of chaotic systems with different dimensions in a certain dimension. Simultaneously, according to the characteristics of the system, a transformation matrix is introduced, which can upgrade or reduce the order of the defined error system to meet the needs of different dimensional systems. Compared with [15-19], the synchronization scheme presented in this paper will not destroy the characteristics of system dimension and will directly synchronize chaotic systems with different dimensions in a certain dimension; on the other hand, the scaling matrix synchronization method used in this paper is not limited to a single synchronization type and can be transformed into a variety of synchronization schemes. In [20-22], although the author further studies the synchronization of chaotic systems with different dimensions by using the scale matrix, most of the results are only applicable to the fully known deterministic chaotic systems. However, they cannot do anything for the uncertain chaotic systems with unknown parameters, and we have done further research on the above work and extended the synchronization problem to the uncertain chaotic or hyperchaotic systems. Because the hyperchaotic system has at least two positive Lyapunov exponents, compared with chaotic system, the higher the degree of instability, the more complex the chaotic behavior. At the same time, according to the characteristics of these systems, appropriate controllers are designed to synchronize and stabilize the chaotic and hyperchaotic systems with different dimensions, which has certain requirements for the design of controllers. These factors bring great difficulties to our research.
This paper is organized as follows. Preliminaries are presented in Section 2. The synchronization theory of different dimension chaotic systems is introduced in detail, and the main conclusions are presented in Section 3. Three examples are presented in Section 4. Finally, in Section 5, concluding comments are given.

\section{Preliminaries}

Consider the following drive system in the form of

$$
\begin{aligned}
\dot{\mathbf{x}} & =F(\mathbf{x}), \\
\mathbf{x}(0) & =\mathbf{x}_{0},
\end{aligned}
$$

where $\mathbf{x} \in \mathbf{R}^{n}$ is the state vector of the drive system and $F(\mathbf{x}): \mathbf{R}^{n} \longrightarrow \mathbf{R}^{n}$ defines a vector field in $n$-dimensional space. The response system is assumed by

$$
\dot{\mathbf{y}}=G(\mathbf{y})+\mathbf{u},
$$

where $\mathbf{y} \in \mathbf{R}^{m}$ is the state vector of the response system, $G(\mathbf{y}): \mathbf{R}^{m} \longrightarrow \mathbf{R}^{m}$ defines a vector field in m-dimensional space, and $\mathbf{u}=\left(u_{1}, \ldots, u_{m}\right)^{T} \in \mathbf{R}^{m}$ is the control input vector.

Definition 1 (see [20]). The drive system (1) and the response system (2) are said to be synchronized in dimension $d$, with respect to scaling matrices $\Theta$ and $\Phi$, if there exists a controller $\mathbf{u} \in \mathbf{R}^{m}$ and given matrices $\Theta \in \mathbf{R}^{d \times m}$ and $\Phi \in \mathbf{R}^{d \times n}$ such that the synchronization error $\mathbf{e}=\Theta \mathbf{y}-\Phi \mathbf{x}$ satisfies that

$$
\lim _{t \longrightarrow \infty}\|\mathbf{e}(t)\|=0,
$$

where $d$ is a certain constant and $\|\cdot\|$ is the vector norm.

In short, if this is the case, then the drive system (1) and the response (2) achieved $\Theta-\Phi$ synchronization.

Remark 1. $\Theta$ and $\Phi$ are constant scaling matrices.

Remark 2 (see [22]). It is easy to see that depending on our choice of the matrices $\Theta$ and $\Phi$, we may have several synchronization types.

(1) The pair $(\Theta, \Phi)=(I, I)$ yields complete synchronization as $\lim _{t \longrightarrow \infty}\|\mathbf{y}(t)-\mathbf{x}(t)\|=0$.

(2) The pair $(\Theta, \Phi)=(I,-I)$ yields antisynchronization as $\lim _{t \longrightarrow \infty}\|\mathbf{y}(t)+\mathbf{x}(t)\|=0$.

(3) The pair $(\Theta, \Phi)=(I, \Lambda), \quad$ where $\Lambda=\operatorname{diag}\left(\lambda_{1}, \lambda_{2}, \ldots, \lambda_{m}\right) \quad\left(\lambda_{i}= \pm 1, i=1, \ldots, m\right)$ yields hybrid synchronization as $\lim _{t \rightarrow \infty}\|\mathbf{y}(t)-\Lambda \mathbf{x}(t)\|=0$.

(4) The pair $(\Theta, \Phi)=(I, \Phi)$ yields matrix projection synchronization as $\lim _{t \rightarrow \infty}\|\mathbf{y}(t)-\Phi \mathbf{x}(t)\|=0$.

Therefore, it can be seen from the above that Definition 1 can be applied not only to the synchronization of chaotic or hyperchaotic systems with different dimensions but also to the synchronization of chaotic or hyperchaotic systems with the same dimensions. 


\section{Scaling Matrix Synchronization of Chaotic Systems with Unknown Parameters}

In this part, our main results include three parts: firstly, considering some unknown parameters in chaotic systems (1) and (2), we design new controller and parameter adaptive laws and give sufficient conditions to ensure the $\Theta-\Phi$ synchronization of chaotic systems with different dimensions; secondly, according to the characteristics of the system model, some new sufficient conditions are given to achieve the desired results; finally, some interesting corollaries are given.

Based on [20], we consider the problem of $\Theta-\Phi$ synchronization between $n$-dimensional drive system and $m$ dimensional response system with unknown parameters.

It is assumed that the drive system and response system with unknown parameters can be written in the following form:

$$
\begin{aligned}
& \dot{\mathbf{x}}=f(\mathbf{x}) \boldsymbol{\alpha}+F_{1}(\mathbf{x}), \\
& \dot{\mathbf{y}}=g(\mathbf{y}) \boldsymbol{\beta}+G_{1}(\mathbf{y})+\mathbf{u},
\end{aligned}
$$

where $\boldsymbol{\alpha}=\left(\alpha_{1}, \alpha_{2}, \ldots, \alpha_{\tau_{1}}\right)^{T}$ are parameters of drive system, $\boldsymbol{\beta}=\left(\beta_{1}, \beta_{2}, \ldots, \beta_{\tau_{2}}\right)^{T}$ are parameters of response system, $\tau_{1}$ and $\tau_{2}$ are constants, and $f: \mathbf{R}^{n} \longrightarrow \mathbf{R}^{n \times \tau_{1}}$, $g: \mathbf{R}^{m} \longrightarrow \mathbf{R}^{m \times \tau_{2}}, F_{1}: \mathbf{R}^{n} \longrightarrow \mathbf{R}^{n}, G_{1}: \mathbf{R}^{m} \longrightarrow \mathbf{R}^{m}$ are all continuous functions.

Considering the error system of Definition 1, then the dynamic equation of the error system is

$$
\dot{\mathbf{e}}=\Theta \dot{\mathbf{y}}-\Phi \dot{\mathbf{x}}
$$

Assumption 1. In general, $0<d \leq \max \{m, n\}$. For the convenience of discussion, we will consider the case $d=m$ of dimension in this paper.

Assumption 2. The matrix $\Theta$ is row full rank matrix.

Remark 3 (see [23]). If the matrix $\Theta$ is row full rank matrix, then define $\Theta^{-1}$ as the right inverse of a matrix $\Theta$.

Since there are unknown parameters in the systems, we assume that $\widehat{\boldsymbol{\alpha}}$ and $\widehat{\boldsymbol{\beta}}$ represent the estimations of $\boldsymbol{\alpha}$ and $\boldsymbol{\beta}$, respectively.

Denote

$$
\begin{aligned}
R_{1}= & (P \Theta \mathbf{y}+\Theta g(\mathbf{y}) \widehat{\boldsymbol{\beta}})-(\Phi f(\mathbf{x}) \widehat{\boldsymbol{\alpha}}+P \Phi \mathbf{x}) \\
& +\Theta G_{1}(\mathbf{y})-\Phi F_{1}(\mathbf{x}),
\end{aligned}
$$

where $\Theta \in \mathbf{R}^{m \times m}$ and $\Phi \in \mathbf{R}^{m \times n}$ are the scaling matrices and $P \in \mathbf{R}^{m \times m}$ is the positive-definite control matrix. For the convenience of calculation, let $P=\operatorname{diag}\left(p_{1}, p_{2}, \ldots, p_{m}\right), p_{i}>0, i=1, \ldots, m$.

The following adaptive laws are proposed to estimate the unknown parameters $\boldsymbol{\alpha}$ and $\boldsymbol{\beta}$ :

$$
\begin{aligned}
\Delta \dot{\boldsymbol{\alpha}} & =(\Phi f(x))^{T} \mathbf{e}-\rho(\Delta \boldsymbol{\alpha}), \\
\Delta \dot{\boldsymbol{\beta}} & =-(\Theta g(\mathbf{y}))^{T} \mathbf{e}-\rho^{\prime}(\Delta \boldsymbol{\beta}),
\end{aligned}
$$

where $\Delta \boldsymbol{\alpha}=\boldsymbol{\alpha}-\widehat{\boldsymbol{\alpha}}, \Delta \boldsymbol{\beta}=\boldsymbol{\beta}-\widehat{\boldsymbol{\beta}}$, and $\rho$ and $\rho^{\prime}$ are the constant gain matrices. For the convenience of calculation, let $\rho=$ $\operatorname{diag}\left(\rho_{1}, \rho_{2}, \ldots, \rho_{\tau_{1}}\right), \rho_{i}>0, i=1, \ldots, \tau_{1} \quad$ and $\rho^{\prime}=\operatorname{diag}\left(\rho_{1}^{\prime}, \rho_{2}^{\prime}, \ldots, \rho_{\tau_{2}}^{\prime}\right), \rho_{i}^{\prime}>0, i=1, \ldots, \tau_{2}$.

Then, based on equation (7), the controller can be designed as follows:

$$
\mathbf{u}=-\Theta^{-1} R_{1} .
$$

Hence, the corresponding error system is obtained as

$$
\begin{aligned}
\dot{\mathbf{e}} & =\Theta \dot{\mathbf{y}}-\Phi \dot{\mathbf{x}} \\
& =\Theta\left(g(\mathbf{y}) \boldsymbol{\beta}+G_{1}(\mathbf{y})+\mathbf{u}\right)-\Phi\left(f(\mathbf{x}) \boldsymbol{\alpha}+F_{1}(\mathbf{x})\right) \\
& =-P \mathbf{e}+\Theta g(\mathbf{y}) \Delta \boldsymbol{\beta}-\Phi f(x) \Delta \boldsymbol{\alpha} .
\end{aligned}
$$

Theorem 1. For the chaotic systems (4) with unknown parameters, if the controller $\mathbf{u}$ is designed as equation (8) and the parameter adaptive laws are designed as equation (7), then the error system (9) is asymptotically stable at the origin. It can also be said that the response system and drive system in (4) achieved $\Theta-\Phi$ synchronization.

Proof. Select the Lyapunov function as follows:

$$
V(t)=\frac{1}{2}\left(\mathbf{e}^{T} \mathbf{e}+(\Delta \boldsymbol{\beta})^{T}(\Delta \boldsymbol{\beta})+(\Delta \boldsymbol{\alpha})^{T}(\Delta \boldsymbol{\alpha})\right) .
$$

Then, the derivative of $V(t)$ along equations (7), (8), and (9) can be calculated as

$$
\begin{aligned}
\dot{V}(t)= & \mathbf{e}^{T} \dot{\mathbf{e}}+(\Delta \boldsymbol{\beta})^{T}(\Delta \dot{\boldsymbol{\beta}})+(\Delta \boldsymbol{\alpha})^{T}(\Delta \dot{\boldsymbol{\alpha}}) \\
= & \mathbf{e}^{T}(-P \mathbf{e}+\Theta g(\mathbf{y}) \Delta \boldsymbol{\beta}-\Phi f(\mathbf{x}) \Delta \boldsymbol{\alpha}) \\
& +(\Delta \boldsymbol{\beta})^{T}\left(-(\Theta g(\mathbf{y}))^{T} \mathbf{e}-\rho^{\prime}(\Delta \boldsymbol{\beta})\right) \\
& +(\Delta \boldsymbol{\alpha})^{T}\left((\Phi f(\mathbf{x}))^{T} \mathbf{e}-\rho(\Delta \boldsymbol{\alpha})\right) . \\
= & -P \mathbf{e}^{T} \mathbf{e}-\rho^{\prime}(\Delta \boldsymbol{\beta})^{T} \Delta \boldsymbol{\beta}-\rho(\Delta \boldsymbol{\alpha})^{T}(\Delta \boldsymbol{\alpha}) .
\end{aligned}
$$

It is clear that $V(t)$ is positive definite and $\dot{V}(t)$ is negative definite in the neighborhood of the zero solution for system (9). According to Lyapunov stability (see Theorem 4.1 in [24]), the synchronous error system (9) is asymptotically stable at the origin. By Definition 1, the synchronization problem of chaotic systems can be transformed into the problem of asymptotic stability of synchronization error system at the origin, so the systems are uniformly asymptotically stable. Therefore, under the action of controller (8) and the parameter adaptive laws (7), the drive system and the response system in (4) achieved $\Theta-\Phi$ synchronization.

This completes the proof of Theorem 1 .

We consider the expression (7) of the parameter adaptive laws in Theorem 1 and transform the constant gain matrix into the function of a gain variable, then the parameter adaptive laws in Theorem 1 can be written as

$$
\begin{aligned}
\Delta \dot{\boldsymbol{\alpha}} & =(\Phi f(\mathbf{x}))^{T} \mathbf{e}-\rho(t)(\Delta \boldsymbol{\alpha}), \\
\Delta \dot{\boldsymbol{\beta}} & =-(\Theta g(\mathbf{y}))^{T} \mathbf{e}-\rho(t)(\Delta \boldsymbol{\beta}),
\end{aligned}
$$

where $\rho(t): \mathbf{R} \longrightarrow \mathbf{R}$ is a gain function. 
The adaptive law of gain function is designed as follows:

$$
\dot{\rho}(t)=(\Delta \boldsymbol{\beta})^{T} \Delta \boldsymbol{\beta}+(\Delta \boldsymbol{\alpha})^{T} \Delta \boldsymbol{\alpha} .
$$

The following Corollary 1 can be obtained.

Corollary 1. For the above chaotic systems (4), keeping the controller in Theorem 1 unchanged and replacing the constant gain matrix with the gain function in the parameter adaptive laws, the system can still achieve $\Theta-\Phi$ synchronization under the action of the controller (8), the parameter adaptive laws (12), and the gain function adaptive law (13).

Proof. The Lyapunov function is selected as

$$
V(t)=\frac{1}{2}\left(\mathbf{e}^{T} \mathbf{e}+(\Delta \boldsymbol{\beta})^{T}(\Delta \boldsymbol{\beta})+(\Delta \boldsymbol{\alpha})^{T}(\Delta \boldsymbol{\alpha})+\rho^{2}(t)\right) .
$$

The proof method is similar to Theorem 1 and omitted.

Let us denote $\alpha_{i}$ or $\beta_{i}$ be the $i$ th unknown parameter of $i$ th equation in the system. Assume that system (4) is linear at unknown parameters $\alpha_{i}$ or $\beta_{i}$. Then, systems (4) can be written as

$$
\begin{aligned}
\dot{\mathbf{x}} & =A_{\mathbf{\alpha}} \mathbf{x}+F_{1}(\mathbf{x}), \\
\dot{\mathbf{y}} & =B_{\beta} \mathbf{y}+G_{1}(\mathbf{y})+\mathbf{v},
\end{aligned}
$$

where $A_{\alpha}$ and $B_{\beta}$ are the diagonal matrix composed of unknown parameters $\alpha$ and $\beta, A_{\alpha}=\operatorname{diag}\left(\alpha_{1}, \ldots, \alpha_{n}\right)$, $B_{\beta}=\operatorname{diag}\left(\beta_{1}, \ldots, \beta_{m}\right)$, and $\mathbf{v}$ is the control input vector.

Note

$$
\widetilde{\mathbf{e}}=\Omega \mathbf{e},
$$

where $\quad \mathbf{e}=\left(e_{1}, \ldots, e_{m}\right)^{T}, \quad \widetilde{\mathbf{e}}=\left(e_{1}, \ldots, e_{n}\right)^{T}, \quad$ and $\Omega=\left(\Omega_{i j}\right) \in \mathbf{R}^{n \times m}$ is the transformation matrix. If $n \leq m$, then $\quad \Omega_{i j}=\left\{\begin{array}{ll}1 & i=j \\ 0 & \text { others }\end{array} ; \quad\right.$ if $n>m, \quad$ then $\Omega_{i j}= \begin{cases}1 & i=j, i=1, \ldots, m \\ 1 & j=m, i=m+1, \ldots, n . \text { Meanwhile, denote } \\ 0 & \text { others }\end{cases}$

$$
\begin{aligned}
R_{2}= & \Theta\left(B_{\beta} \mathbf{y}+G_{1}(\mathbf{y})-B_{\Delta \boldsymbol{\beta}} \mathbf{e}\right) \\
& +K \mathbf{e}+\Phi A_{\Delta \boldsymbol{\alpha}} \widetilde{\mathbf{e}}-\Phi A_{\boldsymbol{\alpha}} \mathbf{x}-\Phi F_{1}(\mathbf{x})
\end{aligned}
$$

where $\quad A_{\Delta \alpha}=A_{\alpha}-A_{\widehat{\alpha}}=\operatorname{diag}\left(\Delta \boldsymbol{\alpha}_{1}, \ldots, \Delta \boldsymbol{\alpha}_{n}\right), \quad B_{\Delta \beta}=B_{\beta}-$ $B_{-}=\operatorname{diag}\left(\Delta \boldsymbol{\beta}_{1}, \ldots, \Delta \boldsymbol{\beta}_{m}\right)$, and $K \in \mathbf{R}^{m \times m}$ is the positivedefinite control matrix; for the convenience of calculation, let $K=\operatorname{diag}\left(k_{1}, k_{2}, \ldots, k_{m}\right), k_{i}>0, i=1, \ldots, m$.

The following new adaptive laws are proposed to estimate the unknown parameters $\alpha$ and $\beta$ :

$$
\begin{aligned}
& \Delta \dot{\boldsymbol{\alpha}}=\operatorname{diag}\left(e_{1}, \ldots, e_{n}\right) \Phi^{T} \mathbf{e}-\psi(\Delta \boldsymbol{\alpha}), \\
& \Delta \dot{\boldsymbol{\beta}}=-\operatorname{diag}\left(e_{1}, \ldots, e_{m}\right) \Theta^{T} \mathbf{e}-\psi^{\prime}(\Delta \boldsymbol{\beta}),
\end{aligned}
$$

where $\psi$ and $\psi^{\prime}$ are the constant gain matrices. For the convenience of calculation, let $\psi=\operatorname{diag}\left(\psi_{1}, \psi_{2}, \ldots \psi_{n}\right), \psi_{i}>0, i=1, \ldots, n \quad$ and $\psi^{\prime}=\operatorname{diag}\left(\psi_{1}^{\prime}, \psi_{2}^{\prime}, \ldots \psi_{m}^{\prime}\right), \psi_{i}^{\prime}>0, i=1, \ldots, m$.

Combined with the adaptive laws of the parameter, the following controller is designed:

$$
\mathbf{v}=-\Theta^{-1} R_{2}
$$

By substituting the controller (19) into the error system (5), it can be obtained:

$$
\begin{aligned}
\dot{\mathbf{e}} & =\Theta \dot{\mathbf{y}}-\Phi \dot{\mathbf{x}} \\
& =\Theta\left(B_{\beta} \mathbf{y}+G_{1}(\mathbf{y})+\mathbf{v}\right)-\Phi\left(A_{\boldsymbol{\alpha}} \mathbf{x}+F_{1}(\mathbf{x})\right) \\
& =\Theta B_{\Delta \beta} \mathbf{e}-\Phi A_{\Delta \boldsymbol{\alpha}} \widetilde{\mathbf{e}}-K \mathbf{e} .
\end{aligned}
$$

Theorem 2. For the chaotic system (15) with unknown parameters, if the controller $\mathbf{v}$ is designed as equation (19) and the parameter adaptive laws are designed as equation (18), then the error system (20) is asymptotically stable at the origin. It can also be said that the response system and drive system in (15) achieved $\Theta-\Phi$ synchronization.

Proof. Select the Lyapunov function as follows:

$$
V(t)=\frac{1}{2}\left(\mathbf{e}^{T} \mathbf{e}+(\Delta \boldsymbol{\beta})^{T}(\Delta \boldsymbol{\beta})+(\Delta \boldsymbol{\alpha})^{T}(\Delta \boldsymbol{\alpha})\right) .
$$

Then, the derivative of $V(t)$ along equations (18), (19), and (20) can be calculated as

$$
\begin{aligned}
\dot{V}(t)= & \mathbf{e}^{T} \dot{\mathbf{e}}+(\Delta \boldsymbol{\beta})^{T}(\Delta \dot{\boldsymbol{\beta}})+(\Delta \boldsymbol{\alpha})^{T}(\Delta \dot{\boldsymbol{\alpha}}) \\
= & \mathbf{e}^{T}\left(\Theta B_{\Delta \boldsymbol{\beta}} \mathbf{e}-\Phi A_{\Delta \boldsymbol{\alpha}} \widetilde{\mathbf{e}}-K \mathbf{e}\right) \\
& +(\Delta \boldsymbol{\beta})^{T}\left(-\operatorname{diag}\left(e_{1}, \ldots, e_{m}\right) \Theta^{T} \mathbf{e}-\psi^{\prime}(\Delta \boldsymbol{\beta})\right) \\
& +(\Delta \boldsymbol{\alpha})^{T}\left(\operatorname{diag}\left(e_{1}, \ldots, e_{n}\right) \Phi^{T} \mathbf{e}-\psi(\Delta \boldsymbol{\alpha})\right) \\
= & -K \mathbf{e}^{T} \mathbf{e}-\psi^{\prime}(\Delta \boldsymbol{\beta})^{T} \Delta \boldsymbol{\beta}-\psi(\Delta \boldsymbol{\alpha})^{T}(\Delta \boldsymbol{\alpha}) .
\end{aligned}
$$

Similar to the proof method of Theorem 1, it is easy to obtain that the error system (20) is asymptotically stable at the origin. By Definition 1, the synchronization problem of chaotic systems can be transformed into the problem of asymptotic stability of synchronization error system at the origin. Therefore, under the joint action of the controller (19) and the parameter adaptive laws (18), the drive system and the response system in (15) achieved $\Theta-\Phi$ synchronization.

This completes the proof of Theorem 2 .

The parameter adaptive laws (18) in Theorem 2 is considered, and the constant gain matrix is transformed into the function of gain variable, and then, the parameter adaptive laws in Theorem 1 can be written as

$$
\begin{aligned}
& \Delta \dot{\boldsymbol{\alpha}}=\operatorname{diag}\left(e_{1}, \ldots, e_{n}\right) \Phi^{T} \mathbf{e}-\psi(t)(\Delta \boldsymbol{\alpha}), \\
& \Delta \dot{\boldsymbol{\beta}}=-\operatorname{diag}\left(e_{1}, \ldots, e_{m}\right) \Theta^{T} \mathbf{e}-\psi(t)(\Delta \boldsymbol{\beta}),
\end{aligned}
$$

where $\psi(t): \mathbf{R} \longrightarrow \mathbf{R}$ is a gain function.

The adaptive law of gain function is designed as follows:

$$
\dot{\psi}(t)=(\Delta \boldsymbol{\beta})^{T} \Delta \boldsymbol{\beta}+(\Delta \boldsymbol{\alpha})^{T} \Delta \boldsymbol{\alpha} .
$$

Corollary 2. For the above chaotic systems (15), if the controller in Theorem 2 remains unchanged and the constant gain matrix is replaced by the gain variable function in the parameter adaptive laws, the systems can still achieve $\Theta-\Phi$ 
synchronization under the joint action of the controller (19), the parameter adaptive laws (23), and the gain function adaptive law (24).

Proof. The Lyapunov function is selected as

$$
V(t)=\frac{1}{2}\left(\mathbf{e}^{T} \mathbf{e}+(\Delta \boldsymbol{\beta})^{T}(\Delta \boldsymbol{\beta})+(\Delta \boldsymbol{\alpha})^{T}(\Delta \boldsymbol{\alpha})+\psi^{2}(t)\right) .
$$

The proof method is similar to Theorem 2 and omitted.

\section{Numerical Simulations}

In this section, we give three examples to verify the effectiveness of our proposed method.

Example 1. Consider the chaotic Cai system [25] as the drive system and the hyperchaotic Rossler system [26] as the response system. The system equations are as follows:

$$
\begin{aligned}
& \left\{\begin{array}{l}
\dot{x}_{1}=a_{1}\left(x_{2}-x_{1}\right), \\
\dot{x}_{2}=b_{1} x_{1}+c_{1} x_{2}-x_{1} x_{3}, \\
\dot{x}_{3}=x_{1}^{2}-d_{1} x_{3},
\end{array}\right. \\
& \left\{\begin{array}{l}
\dot{y}_{1}=-y_{2}-y_{3}+u_{1}, \\
\dot{y}_{2}=y_{1}+a_{2} y_{2}+y_{4}+u_{2}, \\
\dot{y}_{3}=b_{2}+y_{1} y_{3}+u_{3}, \\
\dot{y}_{4}=-c_{2} y_{3}+d_{2} y_{4}+u_{4},
\end{array}\right.
\end{aligned}
$$

where $x_{1}, x_{2}, x_{3}$ are the drive system state variables; $y_{1}, y_{2}, y_{3}, y_{4}$ are the response system state variables; $u_{1}, u_{2}, u_{3}, u_{4}$ are the designed controllers, when the parameters are taken as $a_{1}=20, b_{1}=14, c_{1}=10.6, d_{1}=2.8$ and $a_{2}=0.25, b_{2}=3, c_{2}=0.5, d_{2}=0.05$; and the systems (26) and (27) are chaotic and hyperchaotic. The projection of attractor of chaotic Cai system and hyperchaotic Rossler system is shown in Figures 1 and 2, respectively.
Let make the drive system and the response system achieve synchronization in $4 \mathrm{D}$. We rewrite the systems (26) and (27) in the following forms:

$$
\dot{\mathbf{x}}=f(\mathbf{x}) \boldsymbol{\alpha}+F_{1}(\mathbf{x})
$$

$$
\begin{gathered}
\text { where } f(\mathbf{x})=\left[\begin{array}{cccc}
x_{2}-x_{1} & 0 & 0 & 0 \\
0 & x_{1} & x_{2} & 0 \\
0 & 0 & 0 & -x_{3}
\end{array}\right], \quad \alpha=\left[\begin{array}{l}
a_{1} \\
b_{1} \\
c_{1} \\
d_{1}
\end{array}\right], \quad \text { and } \\
F_{1}(\mathbf{x})=\left[\begin{array}{c}
0 \\
-x_{1} x_{3} \\
x_{1}^{2}
\end{array}\right] . \\
\dot{\mathbf{y}}=g(\mathbf{y}) \boldsymbol{\beta}+G_{1}(\mathbf{y})+\mathbf{u},
\end{gathered}
$$

where $\quad g(\mathbf{y})=\left[\begin{array}{cccc}0 & 0 & 0 & 0 \\ y_{2} & 0 & 0 & 0 \\ 0 & 1 & 0 & 0 \\ 0 & 0 & -y_{3} & y_{4}\end{array}\right], \quad \boldsymbol{\beta}=\left[\begin{array}{c}a_{2} \\ b_{2} \\ c_{2} \\ d_{2}\end{array}\right], \quad G_{1}(\mathbf{x})=$ $\left[\begin{array}{c}-y_{2}-y_{3} \\ y_{1}+y_{4} \\ y_{1} y_{3} \\ 0\end{array}\right]$, and $\mathbf{u}=\left[\begin{array}{l}u_{1} \\ u_{2} \\ u_{3} \\ u_{4}\end{array}\right]$, and we choose

$$
\begin{aligned}
& \Theta=\left[\begin{array}{llll}
1 & 1 & 0 & 0 \\
0 & 1 & 0 & 0 \\
0 & 0 & 1 & 0 \\
0 & 0 & 0 & 1
\end{array}\right], \\
& \Phi=\left[\begin{array}{lll}
1 & 1 & 0 \\
0 & 1 & 0 \\
0 & 1 & 1 \\
1 & 0 & 1
\end{array}\right] .
\end{aligned}
$$

Then, from (6), it can be concluded that

$$
\begin{aligned}
R_{1}= & (P \Theta \mathbf{y}+\Theta g(\mathbf{y}) \widehat{\boldsymbol{\beta}})-(\Phi f(x) \widehat{\boldsymbol{\alpha}}+P \Phi \mathbf{x})+\Theta G_{1}(\mathbf{y})-\Phi F_{1}(\mathbf{x}) \\
= & {\left[\begin{array}{c}
y_{1}+y_{4}+\widehat{a}_{2} y_{2}-\widehat{b}_{1} x_{1}-\widehat{c}_{1} x_{2}+x_{1} x_{3}-y_{2}-y_{3}-\widehat{a}_{1}\left(x_{2}-x_{1}\right)+p_{1} e_{1} \\
y_{1}+y_{4}+\widehat{a}_{2} y_{2}-\widehat{b}_{1} x_{1}-\widehat{c}_{1} x_{2}+x_{1} x_{3}+p_{2} e_{2} \\
y_{1} y_{3}+\widehat{b}_{2}-\widehat{b}_{1} x_{1}-\widehat{c}_{1} x_{2}+x_{1} x_{3}-x_{1}^{2}+\widehat{d}_{1} x_{3}+p_{3} e_{3} \\
-\widehat{c}_{2} y_{3}+\widehat{d}_{2} y_{4}-\widehat{a}_{1}\left(x_{2}-x_{1}\right)-x_{1}^{2}+\widehat{d}_{1} x_{3}+p_{4} e_{4}
\end{array}\right] }
\end{aligned}
$$

From (7), the adaptive law of parameters can be designed as follows:

$$
\left\{\begin{array}{l}
\Delta \dot{a}_{1}=\left(x_{2}-x_{1}\right)\left(e_{1}+e_{4}\right)-\rho_{1}\left(\Delta a_{1}\right) \\
\Delta \dot{b}_{1}=x_{1}\left(e_{1}+e_{2}+e_{3}\right)-\rho_{2}\left(\Delta b_{1}\right) \\
\Delta \dot{c}_{1}=x_{2}\left(e_{1}+e_{2}+e_{3}\right)-\rho_{3}\left(\Delta c_{1}\right) \\
\Delta \dot{d}_{1}=-x_{3}\left(e_{3}+e_{4}\right)-\rho_{4}\left(\Delta d_{1}\right)
\end{array}\right.
$$




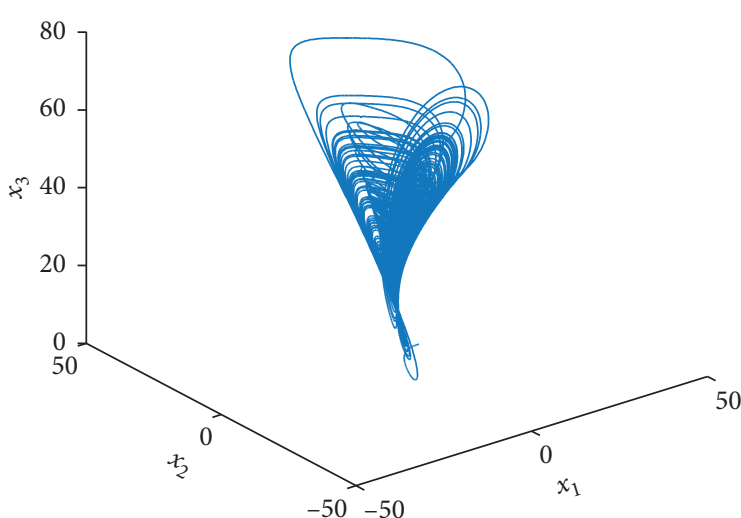

(a)

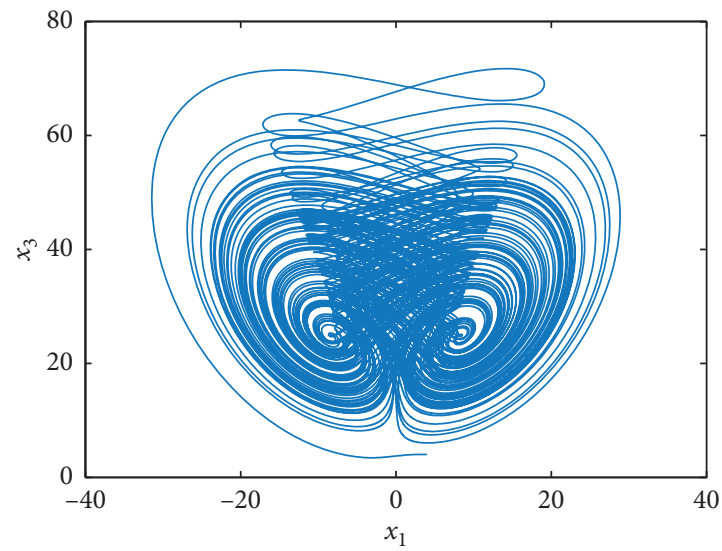

(c)

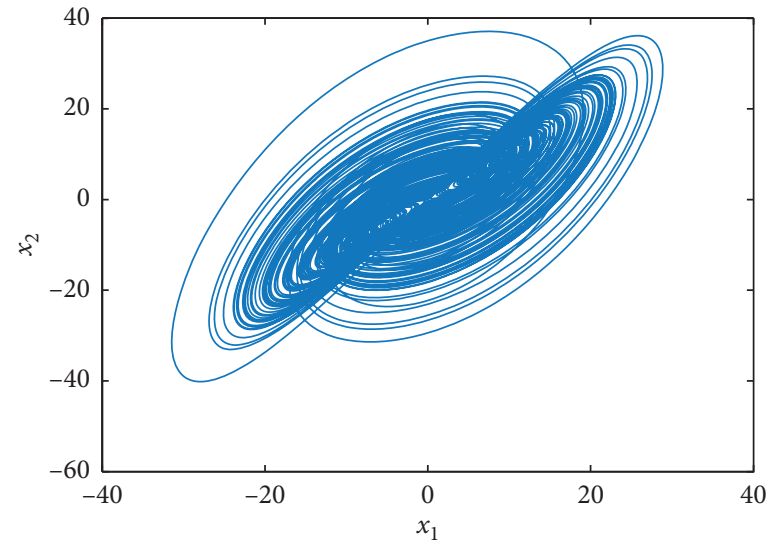

(b)

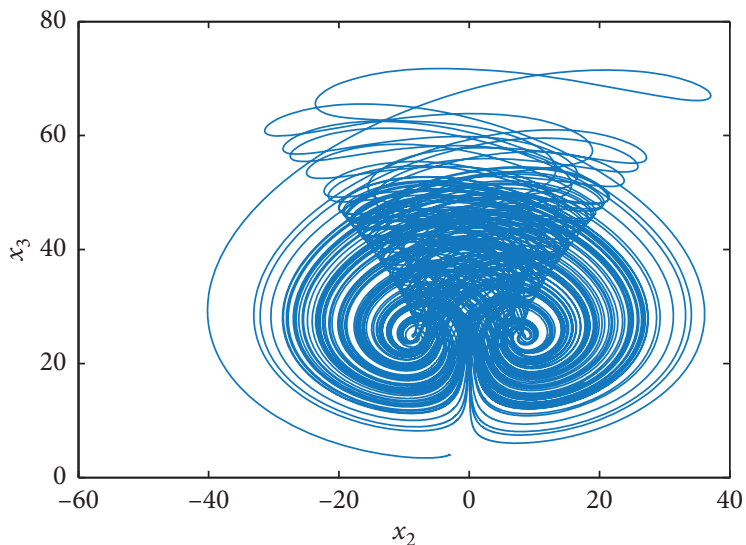

(d)

Figure 1: Typical dynamic behavior of the chaotic Cai system: (a) projection in $\left(x_{1}, x_{2}, x_{3}\right)$ space; (b) projection in $\left(x_{1}, x_{2}\right)$ space; (c) projection in $\left(x_{1}, x_{3}\right)$ space; $(\mathrm{d})$ projection in $\left(x_{2}, x_{3}\right)$ space.

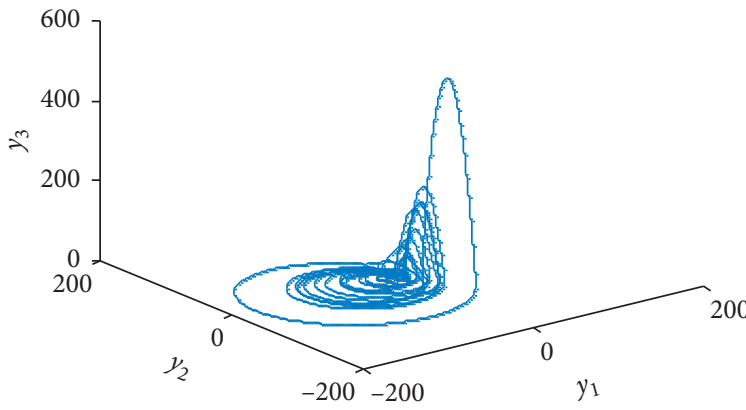

(a)

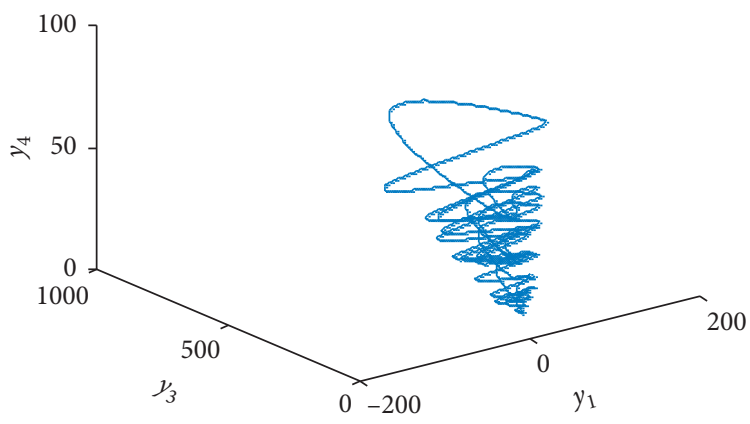

(c)

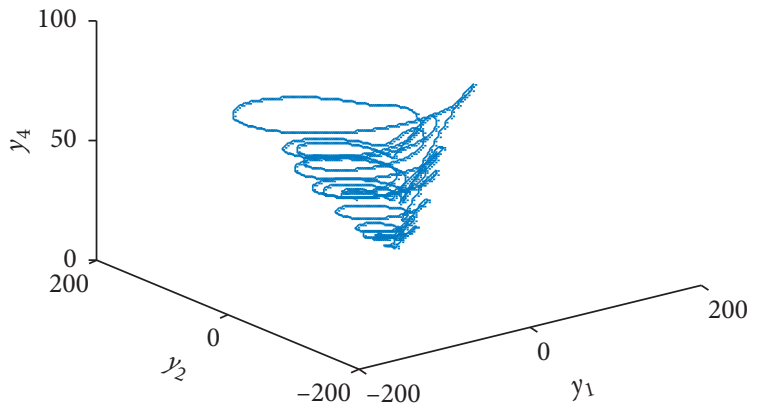

(b)

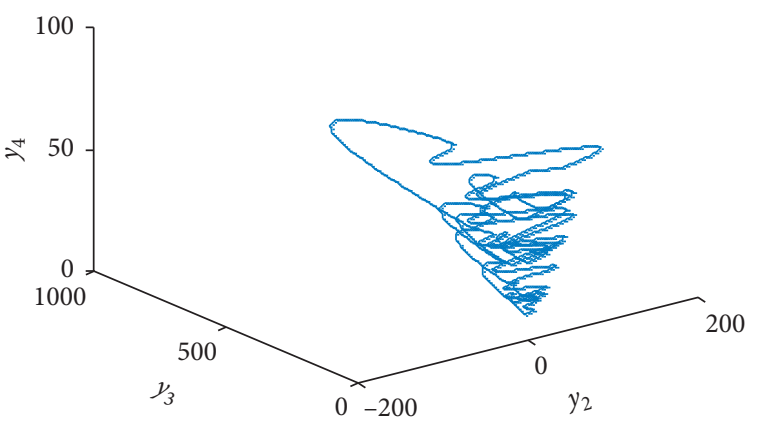

(d)

FIgURe 2: Typical dynamical behaviors of the hyperchaotic Rossler system: (a) projection in $\left(y_{1}, y_{2}, y_{3}\right)$ space; (b) projection in $\left(y_{1}, y_{2}, y_{4}\right)$ space; (c) projection in $\left(y_{1}, y_{3}, y_{4}\right)$ space; (d) projection in $\left(y_{2}, y_{3}, y_{4}\right)$ space. 
From equation (8), the controller can be designed as follows:

$$
\left\{\begin{array}{l}
u_{1}=y_{2}+y_{3}+\widehat{a}_{1}\left(x_{2}-x_{1}\right)-p_{1} e_{1}+p_{2} e_{2}, \\
u_{2}=-y_{1}-y_{4}-\widehat{a}_{2} y_{2}+\widehat{b}_{1} x_{1}+\widehat{c}_{1} x_{2}-x_{1} x_{3}-p_{2} e_{2}, \\
u_{3}=-y_{1} y_{3}-\widehat{b}_{2}+\widehat{b}_{1} x_{1}+\widehat{c}_{1} x_{2}-x_{1} x_{3}+x_{1}^{2}-\widehat{d}_{1} x_{3}-p_{3} e_{3}, \\
u_{4}=\widehat{c}_{2} y_{3}-\widehat{d}_{2} y_{4}+\widehat{a}_{1}\left(x_{2}-x_{1}\right)+x_{1}^{2}-\widehat{d}_{1} x_{3}-p_{4} e_{4} .
\end{array}\right.
$$

In the controller (33), the error system is transformed into

$$
\left\{\begin{array}{l}
\dot{e}_{1}=-\Delta a_{1}\left(x_{2}-x_{1}\right)+\Delta a_{2} y_{2}-\Delta b_{1} x_{1}-\Delta c_{1} x_{2}-p_{1} e_{1} \\
\dot{e}_{2}=\Delta a_{2} y_{2}-\Delta b_{1} x_{1}-\Delta c_{1} x_{2}-p_{2} e_{2} \\
\dot{e}_{3}=\Delta b_{2}-\Delta b_{1} x_{1}-\Delta c_{1} x_{2}+\Delta d_{1} x_{3}-p_{3} e_{3} \\
\dot{e}_{4}=-\Delta c_{2} y_{3}+\Delta d_{2} y_{4}-\Delta a_{1}\left(x_{2}-x_{1}\right)+\Delta d_{1} x_{3}-p_{4} e_{4}
\end{array}\right.
$$

For the numerical simulation, the fourth-order Runge-Kutta integration method has been used to solve the system of differential equations (26) and (27). The initial values of the drive system and response system are $\left(x_{1}(0)\right.$, $\left.x_{2}(0), x_{3}(0)\right)=(4,-3,4)$ and $\left(y_{1}(0), y_{2}(0), y_{3}(0), y_{4}(0)\right)$ $=(5,-6,3,3)$; the initial value of parameter estimations are $\left(\widehat{a}_{1}(0), \widehat{b}_{1}(0), \widehat{c}_{1}(0), \widehat{d}_{1}(0)\right)=(0.1,0.1,0.1,0.1)$ and $\left(\widehat{a}_{2}(0)\right.$, $\left.\widehat{b}_{2}(0), \widehat{c}_{2}(0), \widehat{d}_{2}(0)\right)=(0.1,0.1,0.1,0.1)$. Let two sets of the control matrix and the constant gain matrices be $P=\operatorname{diag}$ $(2,2,2,2), \rho=\operatorname{diag}(1,2,1,2), \rho^{\prime}=\operatorname{diag}(1,1,2,2), \bar{P}=\operatorname{diag}$ $(0.5,0.5,0.5,0.5), \bar{\rho}=\operatorname{diag}(0.2,0.5,0.2,0.5)$, and $\bar{\rho}^{\prime}=\operatorname{diag}$ $(0.2,0.2,0.5,0.5)$, respectively. Figures $3(\mathrm{a})$ and $4(\mathrm{a})$ show the trajectories of errors. It can be seen that the synchronization errors tend to zero; that is, the driving system (26) and the response system (27) achieve stable synchronization in $4 \mathrm{D}$. Figures 3(b), 3(c) and 4(b), 4(c) show the trajectories of the unknown parameter estimations, and it can be seen that the estimations of unknown parameter can converge to some constants.

As can be seen from Figures 3 and 4, if the control constant and gain constant are larger, the convergence speed of synchronization will be faster. The values of control constant and gain constant only affect the speed of synchronization.

$$
\begin{gathered}
P=\operatorname{diag}(2,2,2,2), \\
\rho=\operatorname{diag}(1,2,1,2), \\
\rho^{\prime}=\operatorname{diag}(1,1,2,2) . \\
\bar{P}=\operatorname{diag}(0.5,0.5,0.5,0.5), \\
\bar{\rho}=\operatorname{diag}(0.2,0.5,0.2,0.5), \\
\overline{\rho^{\prime}}=\operatorname{diag}(0.2,0.2,0.5,0.5) .
\end{gathered}
$$

Remark 4. In [15], by adding dimensions to the drive system (26), the synchronization error form $e_{i}=y_{i}-x_{i-1}(i=$ $1,2,3,4)$ of cross subtraction is constructed, and the appropriate controller and parameter adaptive laws are designed so that the drive system (26) and the response system (27) with unknown parameters can be synchronized in $4 D$. By using Theorem 1 and selecting $\Theta=\left[\begin{array}{llll}1 & 0 & 0 & 0 \\ 0 & 1 & 0 & 0 \\ 0 & 0 & 1 & 0 \\ 0 & 0 & 0 & 1\end{array}\right], \Phi=\left[\begin{array}{lll}0 & 0 & 0 \\ 1 & 0 & 0 \\ 0 & 1 & 0 \\ 0 & 0 & 1\end{array}\right]$, $\rho=0, \rho^{\prime}=0$, the controller and parameter adaptive laws designed in this paper are the same as that in [15]; that is, the $\Theta-\Phi$ synchronization in this paper can be transformed into the synchronization scheme in [15]. Compared with the conclusion given in [15], the constant gain matrices and gain function are added to the designed parameter adaptive laws, making Theorem 1 and Corollary 1 given in this paper more universal.

Example 2. Consider the hyperchaotic Lorenz system [27] as the drive system and the chaotic Duffing system [28] as the response system. The system equations are as follows:

$$
\begin{aligned}
& \left\{\begin{array}{l}
x_{1}=\sigma_{1}\left(x_{2}-x_{1}\right), \\
x_{2}=\sigma_{2} x_{1}-\sigma_{3} x_{2}+x_{4}-x_{1} x_{3}, \\
x_{3}=x_{1} x_{2}-\sigma_{4} x_{3}, \\
x_{4}=-\sigma_{5} x_{2}-\sigma_{6} x_{4},
\end{array}\right. \\
& \left\{\begin{array}{l}
\dot{y}_{1}=y_{2}+u_{1}, \\
\dot{y}_{2}=y_{1}-\tau_{1} y_{2}-y_{1}^{3}+\tau_{2} \cos (t)+u_{2},
\end{array}\right.
\end{aligned}
$$

where $x_{1}, x_{2}, x_{3}, x_{4}$ are the drive system state variables; $y_{1}, y_{2}$ are the response system state variables; $u_{1}, u_{2}$ are the designed controllers; and when the parameters are taken as $\sigma_{1}=12, \sigma_{2}=23, \sigma_{3}=1$,

$\sigma_{4}=2.1, \sigma_{5}=6, \sigma_{6}=0.2, \tau_{1}=1, \tau_{2}=0.8$, the systems (37) and (38) are hyperchaotic and chaotic. The projection of attractor of hyperchaotic Lorenz system and chaotic Duffing system is shown in Figures 5 and 6, respectively.

In order to synchronize the drive system and the response system in $2 D$, we rewrite the systems (37) and (38) as follows:

$$
\dot{\mathbf{x}}=f(\mathbf{x}) \boldsymbol{\alpha}+F_{1}(\mathbf{x}),
$$

where $f(\mathbf{x})=\left[\begin{array}{cccc}x_{2}-x_{1} & 0 & 0 & 0 \\ 0 & x_{1} & 0 & 0 \\ 0 & 0 & -x_{3} & 0 \\ 0 & 0 & 0 & -x_{4}\end{array}\right], \boldsymbol{\alpha}=\left[\begin{array}{c}\sigma_{1} \\ \sigma_{2} \\ \sigma_{3} \\ \sigma_{4}\end{array}\right]$, and $F_{1}$ $(\mathbf{x})=\left[\begin{array}{c}x_{4} \\ -x_{2}-x_{1} x_{3} \\ x_{1} x_{2} \\ -x_{2} x_{3}\end{array}\right]$

$$
\dot{\mathbf{y}}=g(\mathbf{y}) \boldsymbol{\beta}+G_{1}(\mathbf{y}),
$$




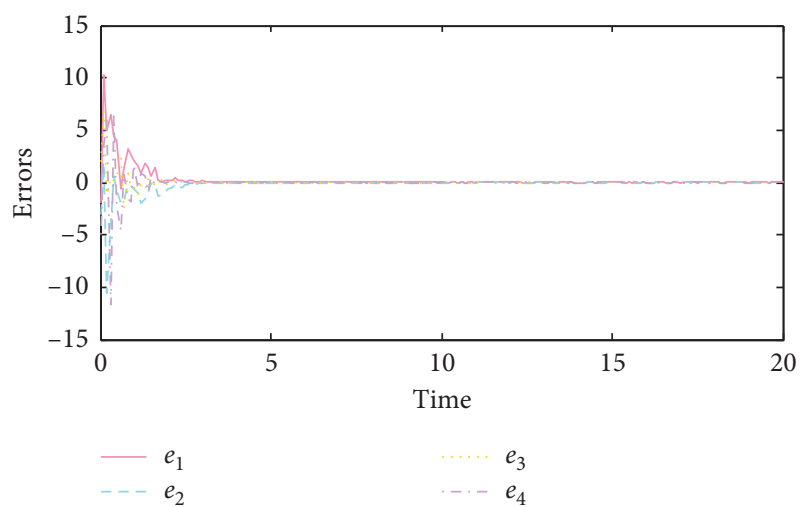

(a)

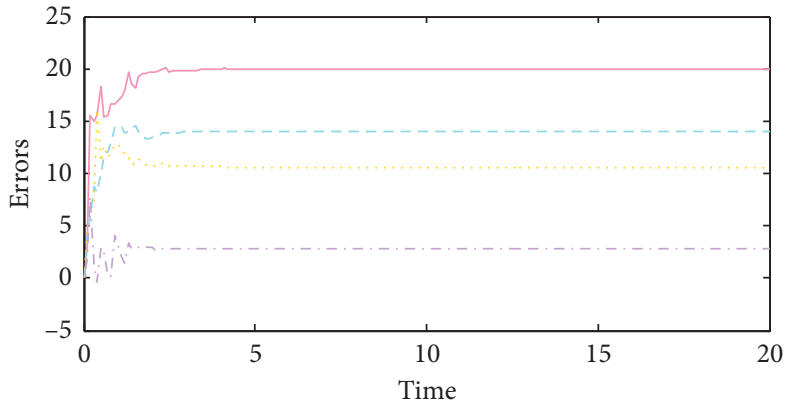

$\begin{array}{ll}- & \hat{a}_{1} \\ --- & \hat{b}_{1}\end{array}$

(b)

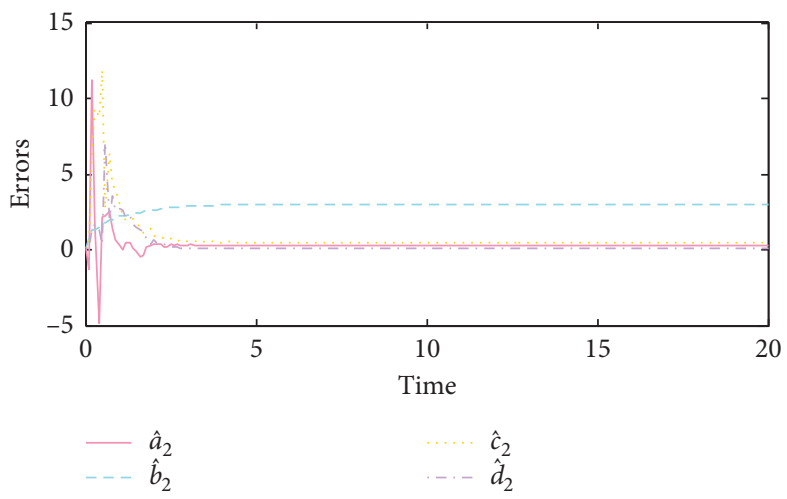

(c)

Figure 3: (a) Trajectories of the errors; (b) trajectories of the drive system unknown parameter estimates; (c) trajectories of the response system unknown parameter estimates.

where $\quad g(\mathbf{y})=\left[\begin{array}{cc}0 & 0 \\ -y_{2} & \cos t\end{array}\right], \boldsymbol{\beta}=\left[\begin{array}{l}\tau_{1} \\ \tau_{2}\end{array}\right]$, and $G_{1}(\mathbf{x})=$ $\left[\begin{array}{c}y_{2} \\ y_{1}-y_{1}^{3}\end{array}\right]$, and we choose

$\Theta=\left[\begin{array}{ll}2 & 0 \\ 0 & 1\end{array}\right], \Phi=\left[\begin{array}{llll}1 & 0 & 1 & 0 \\ 0 & 1 & 0 & 1\end{array}\right]$.

$$
\begin{aligned}
R_{1} & =(P \Theta \mathbf{y}+\Theta g(\mathbf{y}) \widehat{\boldsymbol{\beta}})-(\Phi f(x) \widehat{\boldsymbol{\alpha}}+P \Phi \mathbf{x})+\Theta G_{1}(\mathbf{y})-\Phi F_{1}(\mathbf{x}) \\
& =\left[\begin{array}{c}
2 y_{2}-x_{1} x_{2}-\widehat{\sigma}_{1}\left(x_{2}-x_{1}\right)+\widehat{\sigma}_{3} x_{3}+p_{1} e_{1} \\
y_{1}-y_{1}^{3}-x_{4}+x_{3} x_{1}-\widehat{\tau}_{1} y_{2}+\widehat{\tau}_{2} \cos t-\widehat{\sigma}_{2} x_{1}+\widehat{\sigma}_{3} x_{2}+\widehat{\sigma}_{5} x_{2}+\widehat{\sigma}_{6} x_{4}+p_{2} e_{2}
\end{array}\right] .
\end{aligned}
$$




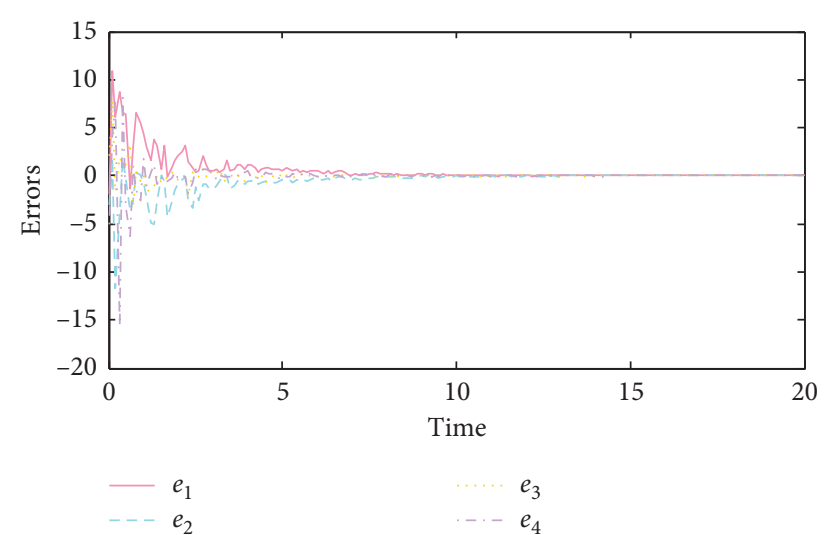

(a)

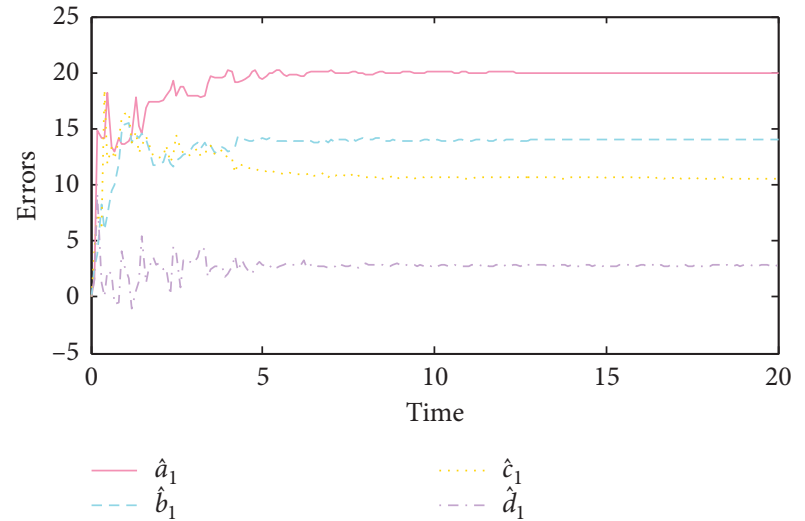

(b)

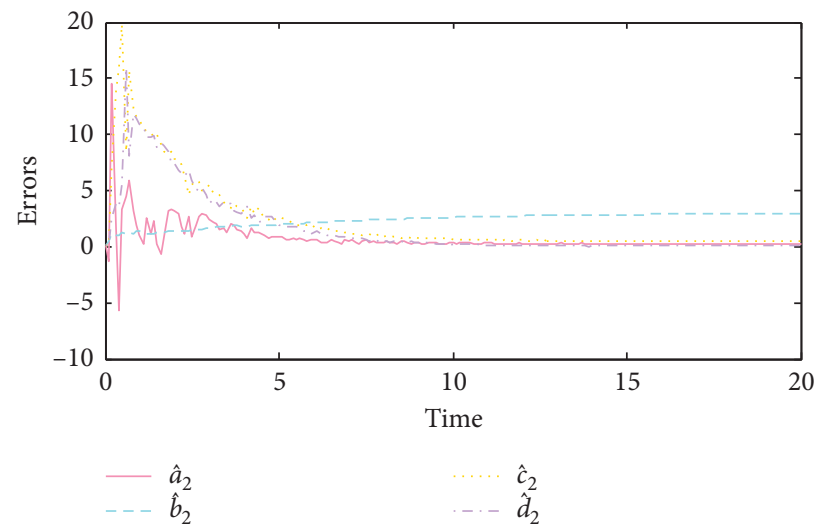

(c)

FiguRE 4: (a) Trajectories of the errors; (b) trajectories of the drive system unknown parameter estimates; (c) trajectories of the response system unknown parameter estimates.

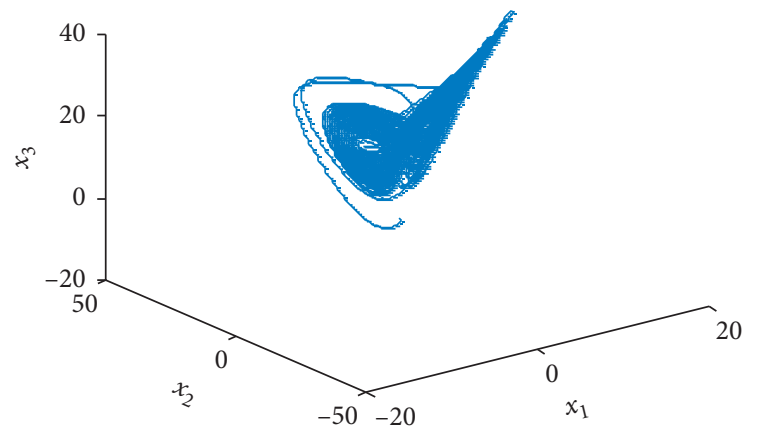

(a)

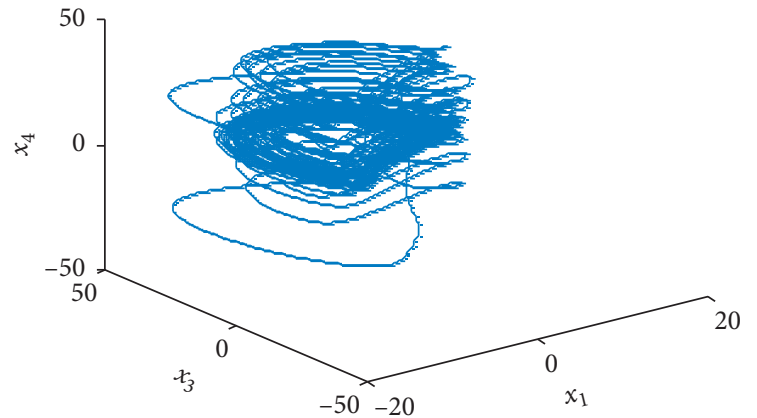

(c)

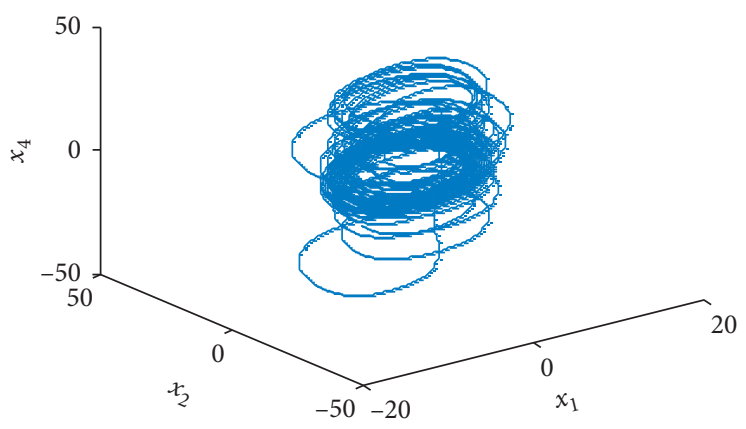

(b)

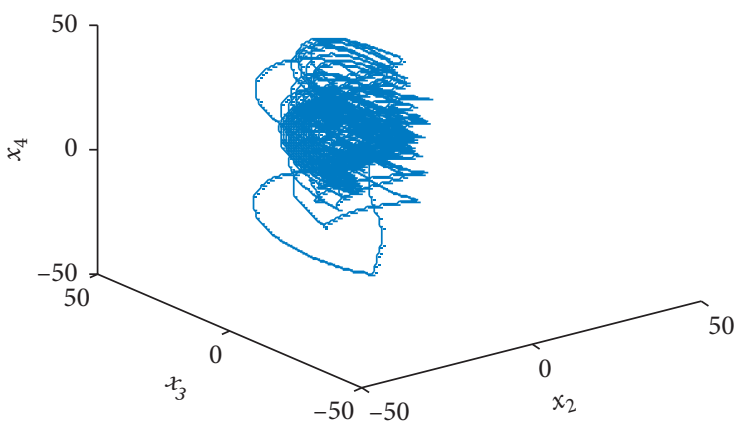

(d)

FIGURE 5: Typical dynamical behaviors of the hyperchaotic Lorenz system: (a) projection in $\left(x_{1}, x_{2}, x_{3}\right)$ space; (b) projection in $\left(x_{1}, x_{2}, x_{4}\right)$ space; (c) projection in $\left(x_{1}, x_{3}, x_{4}\right)$ space; (d) projection in $\left(x_{2}, x_{3}, x_{4}\right)$ space. 


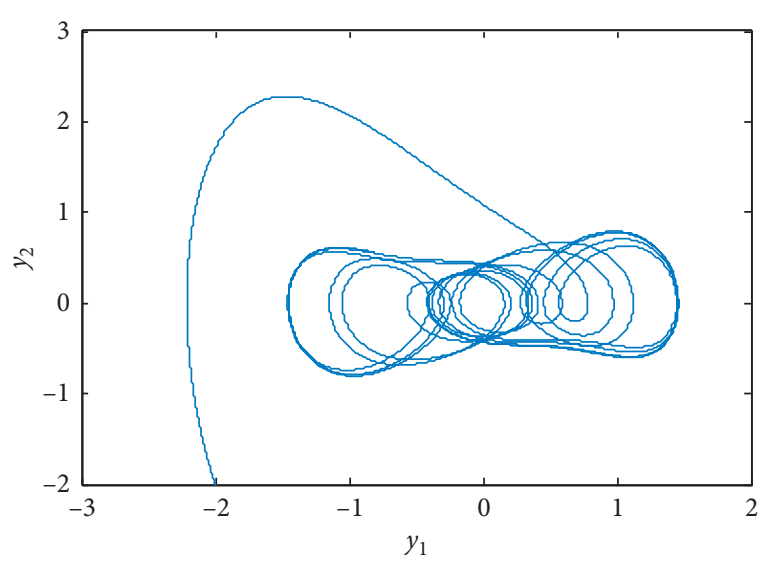

FIGURe 6: Typical dynamic behavior of the chaotic Duffing system: projection in $\left(y_{1}, y_{2}\right)$ space.

From (12) and (13), the adaptive law of parameters can be designed as follows:

$$
\begin{aligned}
& \left\{\begin{array}{l}
\Delta \dot{\sigma}_{1}=\left(x_{2}-x_{1}\right) e_{1}-\rho(t), \\
\Delta \sigma_{2}=x_{1} e_{2}-\rho(t), \\
\Delta \sigma_{3}=-x_{2} e_{2}-\rho(t), \\
\Delta \sigma_{4}=-x_{3} e_{1}-\rho(t), \\
\Delta \sigma_{3}=-x_{2} e_{2}-\rho(t), \\
\Delta \sigma_{4}=-x_{4} e_{2}-\rho(t),
\end{array}\right. \\
& \left\{\begin{array}{l}
\Delta \dot{\tau}_{1}=e_{2} y_{2}-\rho(t), \\
\Delta \dot{\tau}_{2}=-e_{2} \cos t-\rho(t),
\end{array}\right.
\end{aligned}
$$

where

$$
\dot{\rho}(t)=\Delta \sigma_{1}^{2}+\Delta \sigma_{2}^{2}+\Delta \sigma_{3}^{2}+\Delta \sigma_{4}^{2}+\Delta \sigma_{5}^{2}+\Delta \sigma_{6}^{2}+\Delta \tau_{1}^{2}+\Delta \tau_{2}^{2}
$$

From equation (8), the controller can be designed as follows:

$$
\left\{\begin{array}{l}
u_{1}=-y_{2}+\frac{1}{2} x_{1} x_{2}+\frac{1}{2} \widehat{\sigma}_{1}\left(x_{2}-x_{1}\right)-\frac{1}{2} \widehat{\sigma}_{4} x_{3}-\frac{1}{2} p_{1} e_{1} \\
u_{2}=-y_{1}+y_{1}^{3}+x_{4}-x_{1} x_{3}+\widehat{\tau}_{1} y_{2}-\widehat{\tau}_{2} \cos t+\widehat{\sigma}_{2} x_{1}-\widehat{\sigma}_{3} x_{2}-\widehat{\sigma}_{5} x_{2}-\widehat{\sigma}_{6} x_{4}-p_{2} e_{2}
\end{array}\right.
$$
into

In the controller (45), the error system is transformed $\left\{\begin{array}{l}\dot{e}_{1}=-\Delta \sigma_{1}\left(x_{2}-x_{1}\right)+\Delta \sigma_{4} x_{3}-p_{1} e_{1}, \\ \dot{e}_{2}=-\Delta \tau_{1} y_{2}+\Delta \tau_{2} \cos t-\Delta \sigma_{2} x_{1}+\Delta \sigma_{3} x_{2}+\Delta \sigma_{5} x_{2}+\Delta \sigma_{6} x_{4}-p_{2} e_{2} .\end{array}\right.$

For numerical simulation, the fourth-order Runge-Kutta integration method has been used to solve the system of differential (37) and (38). The initial values of the drive system and response system are $\left(x_{1}(0), x_{2}(0), x_{3}(0), x_{4}(0)\right)=(-1$, $-0.6,-1,-0.2)$ and $\left(y_{1}(0), y_{2}(0)\right)=(-2,-2)$, respectively, and the initial value of parameter estimations is $\left(\widehat{\sigma}_{1}(0), \widehat{\sigma}_{2}\right.$ $\left.(0), \widehat{\sigma}_{3}(0), \widehat{\sigma}_{4}(0), \widehat{\sigma}_{5}(0), \widehat{\sigma}_{6}(0)\right)=(10,20,3,2,1,2)$ and $\left(\widehat{\tau}_{1}\right.$ (0), $\left.\widehat{\tau}_{2}(0)\right)=(0.1,0.2)$, respectively; the control matrix $P=$ $\operatorname{diag}(3,2)$, and the initial value of the gain function $\rho(0)=$ 0.1 . Figure 7 (a) shows the trajectories of errors, and it can be seen that the synchronization errors tend to zero; that is, the driving system (37) and the response system (48) achieve stable synchronization in $2 D$. Figures 7(b) and 7(c) show the trajectories of the unknown parameter estimations, and it can be seen that the estimations of the unknown parameter can converge to some constants.

Remark 5. In [23], the global fixed-time synchronization of four-dimensional hyperchaotic system (37) and two-dimensional chaotic Duffing system (38) is studied. By using Theorem 1 and selecting $\Theta=\left[\begin{array}{cc}-1 & 0 \\ 0 & -1\end{array}\right], \Phi=\left[\begin{array}{cccc}-1 & -1 & 0 & 0 \\ 0 & 0 & -1 & -1\end{array}\right], \rho=$
$0, \rho^{\prime}=0$, the synchronization type in this paper is the same as that in [23]. However, the authors in [23] focus on the problem of global fixed-time synchronization. When estimating unknown parameters, due to many constraints, the controller designed in [23] is more complex and difficult to meet in practice. Compared with the controller given in [23], the controller designed in this paper is relatively simple. At the same time, compared with the parameter adaptive laws given in [23], the constant gain matrices and the gain function are added in the designed parameter adaptive laws, which make the parameter estimation function given in this paper more effective.

Example 3. Consider the chaotic financial system [29] as the drive system and the hyperchaotic Chen-Lee system [30] as the response system. The system equations are as follows:

$$
\begin{gathered}
\left\{\begin{array}{l}
\dot{x}_{1}=x_{3}+\left(x_{2}-r_{1}\right) x_{1}, \\
\dot{x}_{2}=1-r_{2} x_{2}-x_{1}^{2}, \\
\dot{x}_{3}=-x_{1}-r_{3} x_{3},
\end{array}\right. \\
\left\{\begin{array}{l}
\dot{y}_{1}=\omega_{1} y_{1}-y_{2} y_{3}+v_{1}, \\
\dot{y}_{2}=\omega_{2} y_{2}+y_{1} y_{3}+v_{2}, \\
\dot{y}_{3}=\omega_{3} y_{3}+0.2 y_{4}+\frac{1}{3} y_{1} y_{2}+v_{3}, \\
\dot{y}_{4}=2.2 y_{1}+0.05 y_{4}+0.5 y_{2} y_{3}+v_{4},
\end{array}\right.
\end{gathered}
$$




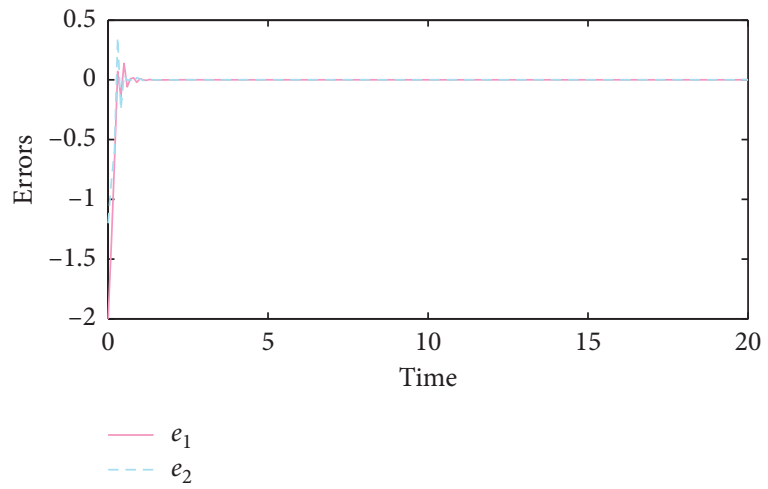

(a)

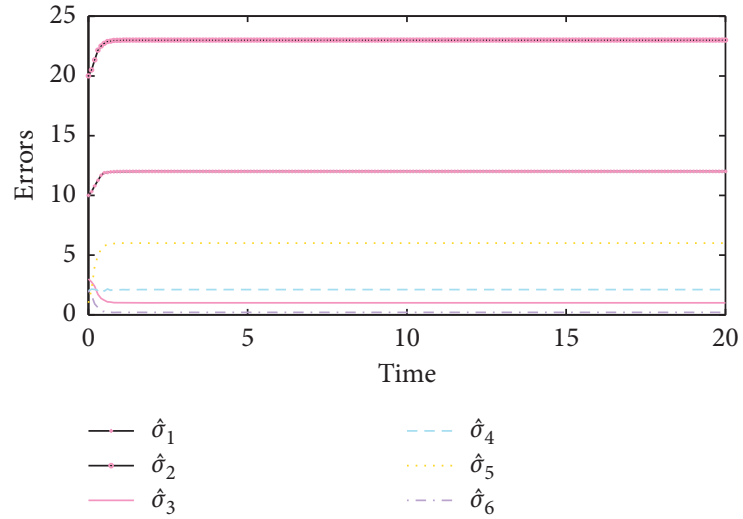

(b)

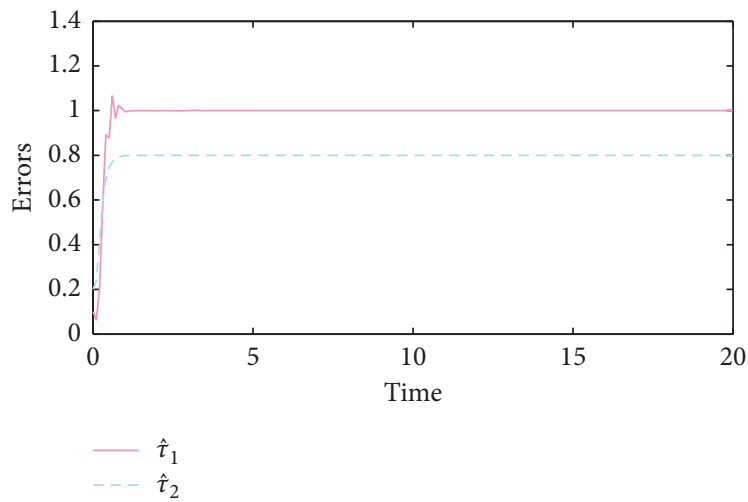

(c)

Figure 7: (a) Trajectories of the errors; (b) trajectories of the drive system unknown parameter estimates; (c) trajectories of the response system unknown parameter estimates.

where $x_{1}, x_{2}, x_{3}$ are the drive system state variables, $y_{1}, y_{2}$, $y_{3}, y_{4}$ are the response system state variables, and $v_{1}, v_{2}, v_{3}$, $v_{4}$ are the designed controllers. When the parameters are taken as $r_{1}=0.8, r_{2}=0.2, r_{3}=1.9, \omega_{1}=5, \omega_{2}=-10, \omega_{3}=$ -3.8 , the systems (47) and (48) are chaotic and hyperchaotic. The projection of attractor of chaotic financial system and hyperchaotic Chen-Lee system is shown in Figures 8 and 9, respectively.

In order to synchronize the drive system and the response system in $4 D$, we rewrite the systems (47) and (48) as follows:

$$
\dot{\mathbf{x}}=A_{\alpha} \mathbf{x}+F_{1}(\mathbf{x})
$$

where $A_{\alpha}=\left[\begin{array}{ccc}r_{1} & 0 & 0 \\ 0 & r_{2} & 0 \\ 0 & 0 & r_{3}\end{array}\right]$ and $F_{1}(\mathbf{x})=\left[\begin{array}{c}x_{3}+x_{1} x_{2} \\ 1-x_{1}^{2} \\ -x_{1}\end{array}\right]$.

$$
\dot{\mathbf{y}}=B_{\beta} \mathbf{y}+G_{1}(\mathbf{y})+\mathbf{v}
$$

$$
\begin{aligned}
& \text { where } \quad B_{\beta}=\left[\begin{array}{cccc}
\omega_{1} & 0 & 0 & 0 \\
0 & \omega_{2} & 0 & 0 \\
0 & 0 & \omega_{3} & 0 \\
0 & 0 & 0 & 0
\end{array}\right] \text { and } G_{1}(\mathbf{y})= \\
& {\left[\begin{array}{c}
-y_{2} y_{3} \\
y_{1} y_{3} \\
0.2 y_{4}+1 / 3 y_{1} y_{2} \\
2.2 y_{1}+0.05 y_{4}+0.5 y_{2} y_{3}
\end{array}\right] \text {, and we choose }}
\end{aligned}
$$

$$
\begin{gathered}
\Theta=\left[\begin{array}{llll}
1 & 0 & 0 & 0 \\
0 & 1 & 0 & 0 \\
0 & 0 & 1 & 0 \\
1 & 0 & 0 & 1
\end{array}\right], \\
\Phi=\left[\begin{array}{lll}
1 & 0 & 0 \\
0 & 1 & 0 \\
0 & 0 & 1 \\
1 & 1 & 0
\end{array}\right] .
\end{gathered}
$$

Then, from (17), it can be concluded that 

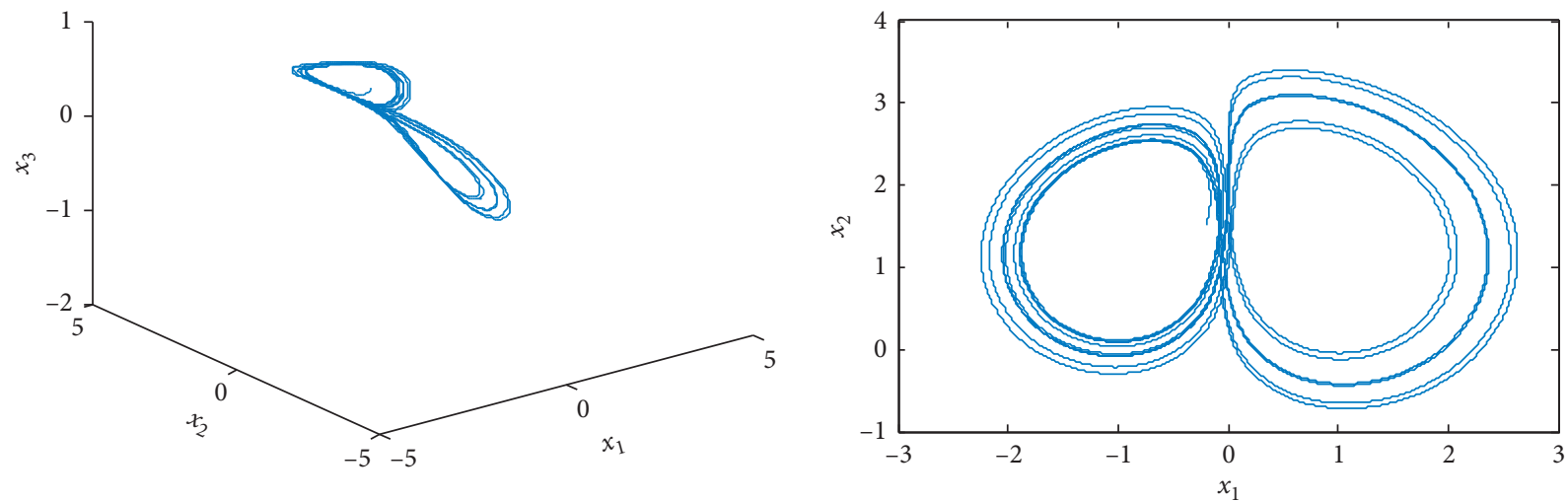

(a)

(b)

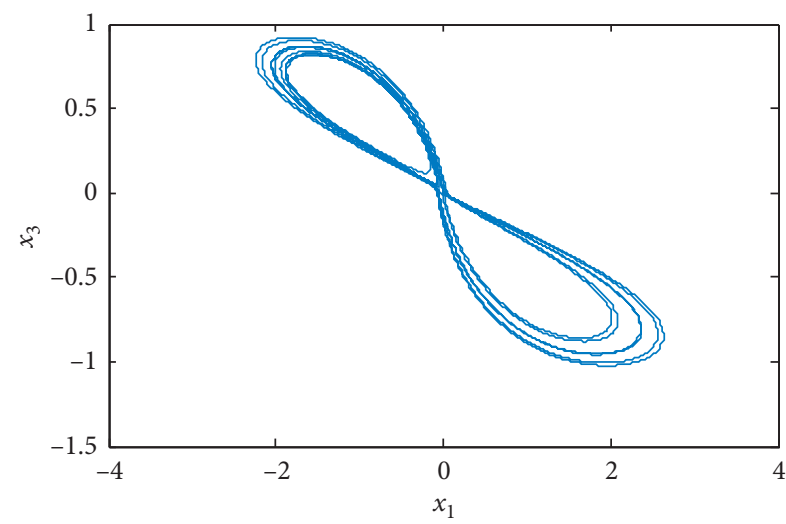

(c)

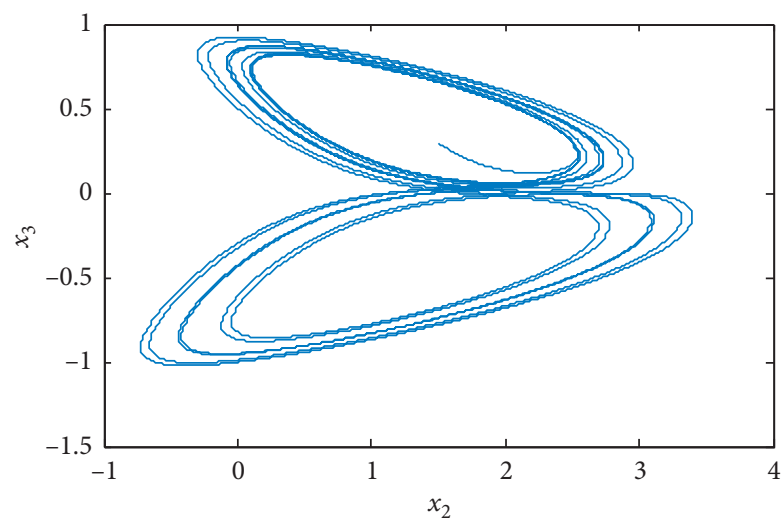

(d)

Figure 8: Typical dynamic behavior of the chaotic financial system: (a) projection in $\left(x_{1}, x_{2}, x_{3}\right)$ space; (b) projection in $\left(x_{1}, x_{2}\right)$ space; (c) projection in $\left(x_{1}, x_{3}\right)$ space; $(\mathrm{d})$ projection in $\left(x_{2}, x_{3}\right)$ space.

$$
\begin{aligned}
R_{2}= & \Theta\left(B_{\boldsymbol{\beta}} \mathbf{y}+G_{1}(\mathbf{y})-B_{\Delta \boldsymbol{\beta}} \mathbf{e}\right)+K \mathbf{e}+\Phi A_{\Delta \mathbf{\alpha}} \widetilde{\mathbf{e}}-\Phi A_{\mathbf{\alpha}} \mathbf{x}-\Phi F_{1}(\mathbf{x}) \\
= & {\left[\begin{array}{c}
\omega_{1} x_{1}+r_{1} y_{1}-y_{2} y_{3}-x_{2} x_{1}-x_{3}+\widehat{\omega}_{1} e_{1}-\widehat{r}_{1} e_{1}+k_{1} e_{1} \\
y_{1} y_{3}-1+x_{1}^{2}+\omega_{2} x_{2}+r_{2} y_{2}+\widehat{\omega}_{2} e_{2}-\widehat{r}_{2} e_{2}+k_{2} e_{2} \\
0.2 y_{4}+\frac{1}{3} y_{1} y_{2}+x_{1}+\omega_{3} x_{3}+r_{3} y_{3}+\widehat{\omega}_{3} e_{2}-\widehat{r}_{3} e_{2}+k_{3} e_{3} \\
\omega_{1} x_{1}+r_{1} y_{1}+\widehat{\omega}_{1} e_{1}-\widehat{r}_{1} e_{1}+k_{1} e_{1}-y_{2} y_{3}-x_{2} x_{1} \\
-x_{3}+2.2 y_{1}+0.05 y_{4}+0.5 y_{2} y_{3}-1+x_{1}^{2}-y_{2} y_{3} \\
-x_{2} x_{1}-x_{3}+\omega_{1} x_{1}+r_{1} y_{1}+\widehat{\omega}_{1} e_{1}-\widehat{r}_{1} e_{1}+r_{2} y_{2}-\widehat{r}_{2} e_{2}+k_{4} e_{4}
\end{array}\right] . }
\end{aligned}
$$

From equation (18), the adaptive law of parameters can be designed as follows:

$$
\left\{\begin{array}{l}
\Delta \dot{r}_{1}=e_{1}^{2}+e_{1} e_{4}-\psi_{1}\left(\Delta r_{1}\right), \\
\Delta \dot{r}_{2}=e_{2}^{2}+e_{2} e_{4}-\psi_{2}\left(\Delta r_{2}\right), \\
\Delta \dot{r}_{3}=e_{3}^{2}-\psi_{3}\left(\Delta r_{3}\right),
\end{array}\right.
$$

$$
\left\{\begin{array}{l}
\Delta \dot{\omega}_{1}=-e_{1}^{2}-e_{1} e_{4}-\psi_{1}^{\prime}\left(\Delta \omega_{1}\right), \\
\Delta \dot{\omega}_{2}=-e_{2}^{2}-\psi_{2}^{\prime}\left(\Delta \omega_{2}\right), \\
\Delta \dot{\omega}_{3}=-e_{3}^{2}-\psi_{3}^{\prime}\left(\Delta \omega_{2}\right) .
\end{array}\right.
$$

From equation (19), the controller can be designed as follows:

$$
\left\{\begin{array}{l}
v_{1}=y_{2} y_{3}+x_{2} x_{1}+x_{3}-\omega_{1} x_{1}-r_{1} y_{1}-\widehat{\omega}_{1} e_{1}+\widehat{r}_{1} e_{1}-k_{1} e_{1}, \\
v_{2}=-y_{1} y_{3}+1-x_{1}^{2}-\omega_{2} x_{2}-r_{2} y_{2}-\widehat{\omega}_{2} e_{2}+\widehat{r}_{2} e_{2}-k_{2} e_{2} \\
v_{3}=-0.2 y_{4}-\frac{1}{3} y_{1} y_{2}-x_{1}-\omega_{3} x_{3}-r_{3} y_{3}-\widehat{\omega}_{3} e_{3}+\widehat{r}_{3} e_{3}-k_{3} e_{3}, \\
v_{4}=-2.2 y_{1}-0.05 y_{4}-0.5 y_{2} y_{3}+1-x_{1}^{2}-r_{2} y_{2}+\widehat{r}_{2} e_{2}-k_{4} e_{4} .
\end{array}\right.
$$

In the controller (54), the error system is transformed into 


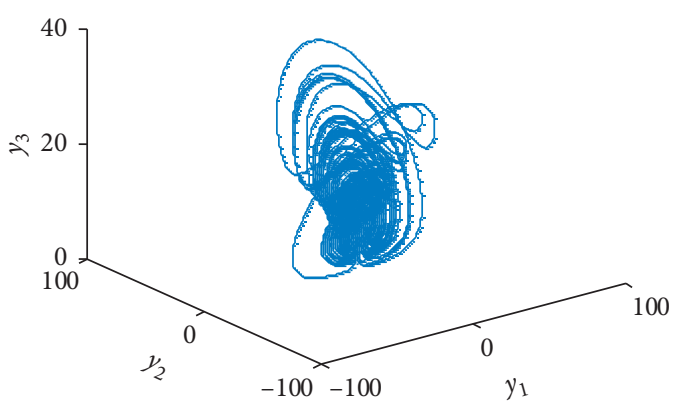

(a)

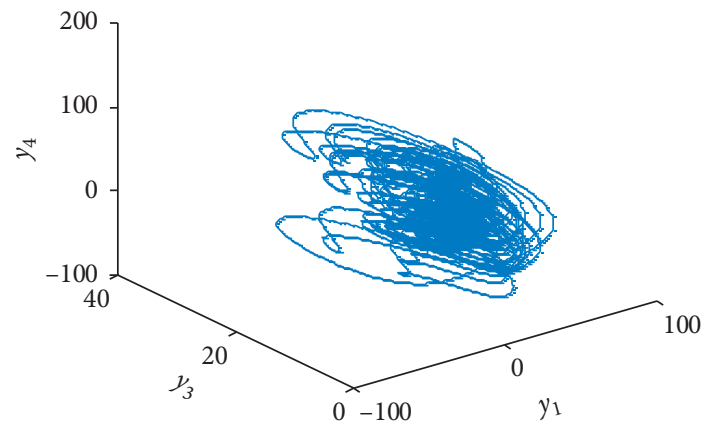

(c)

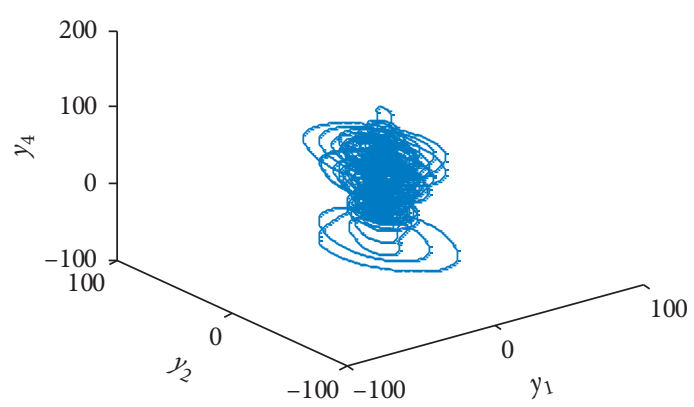

(b)

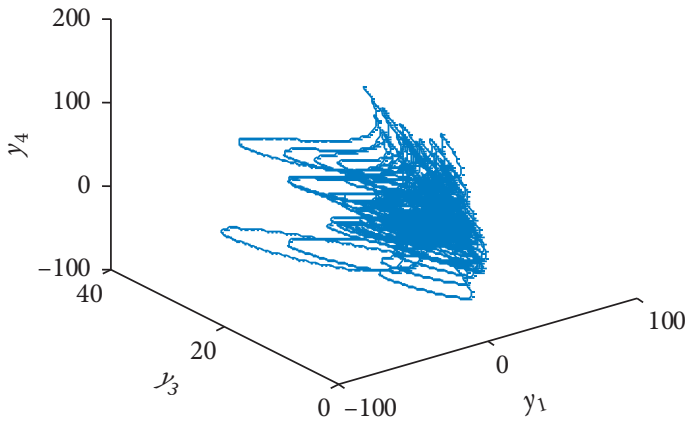

(d)

Figure 9: Typical dynamical behaviors of the hyperchaotic Chen-Lee system: (a) projection in $\left(y_{1}, y_{2}, y_{3}\right)$ space; (b) projection in $\left(y_{1}, y_{2}, y_{4}\right)$ space; (c) projection in $\left(y_{1}, y_{3}, y_{4}\right)$ space; (d) projection in $\left(y_{2}, y_{3}, y_{4}\right)$ space.

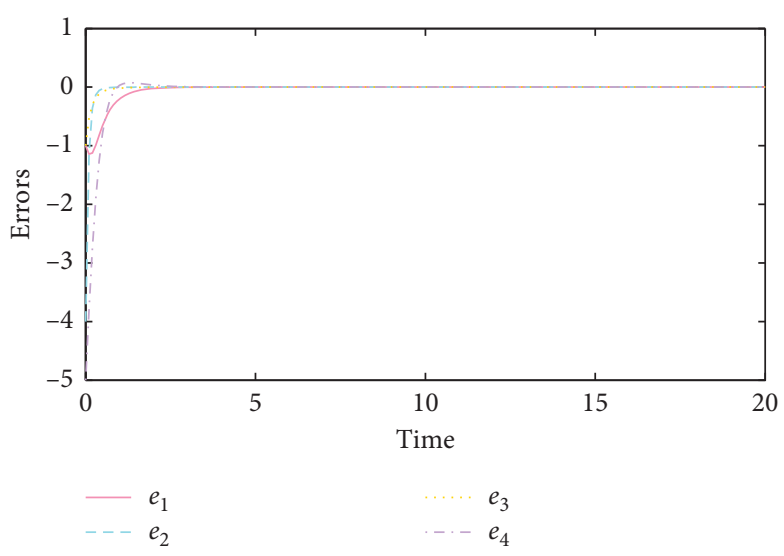

(a)

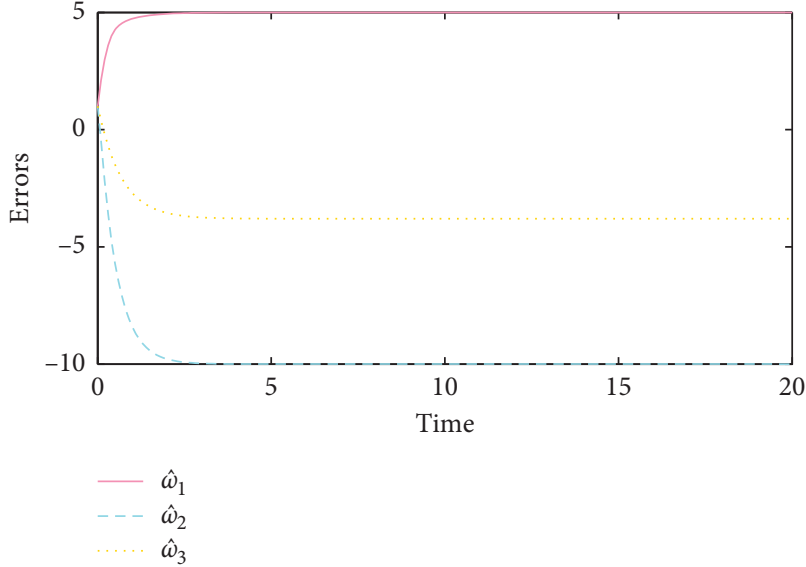

(b)

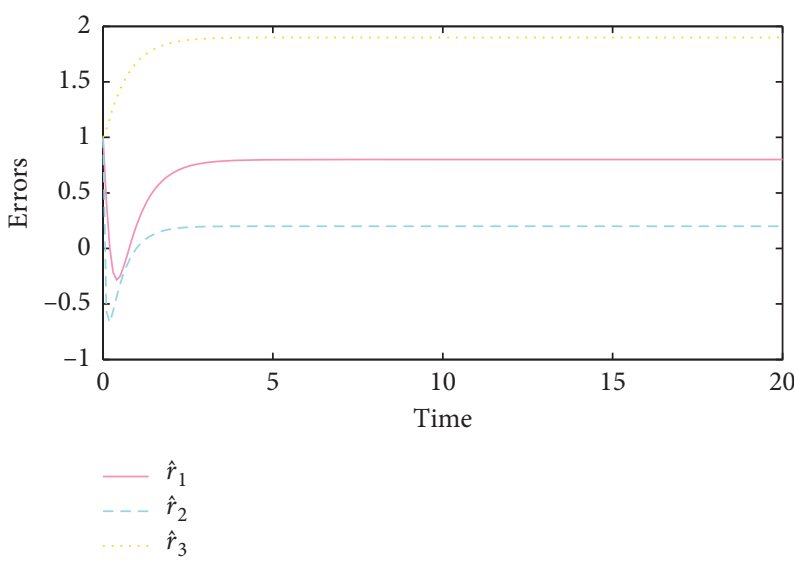

(c)

Figure 10: (a) Trajectories of the errors: (b) trajectories of the drive system unknown parameter estimates; (c) trajectories of the response system unknown parameter estimates. 


$$
\left\{\begin{array}{l}
\dot{e}_{1}=\left(\Delta \omega_{1}-\Delta r_{1}\right) e_{1}-k_{1} e_{1}, \\
\dot{e}_{2}=\left(\Delta \omega_{2}-\Delta r_{2}\right) e_{2}-k_{2} e_{2}, \\
\dot{e}_{3}=\left(\Delta \omega_{3}-\Delta r_{3}\right) e_{3}-k_{2} e_{3}, \\
\dot{e}_{4}=\left(\Delta \omega_{1}-\Delta r_{1}\right) e_{1}-\Delta r_{2} e_{2}-k_{4} e_{4} .
\end{array}\right.
$$

For numerical simulation, the fourth-order Runge-Kutta integration method has been used to solve the system of differential (47) and (48). The initial values of the drive system and response system are $\left(x_{1}(0), x_{2}(0), x_{3}(0)\right)$ $=(2,6,4)$ and $\left(y_{1}(0), y_{2}(0), y_{3}(0), y_{4}(0)\right)=(1,2,3,2)$, respectively; the initial value of parameter estimations is $\left(\widehat{r}_{1}(0), \widehat{r}_{2}(0), \widehat{r}_{3}(0)\right)=(0.1,0.1,0.1)$ and $\left(\widehat{\omega}_{1}(0), \widehat{\omega}_{2}(0), \widehat{\omega}_{3}\right.$ $(0))=(0.1,0.1,0.1)$, the control matrix $K=\operatorname{diag}(2,2,1,3)$, and the constant gain matrices $\psi=\operatorname{diag}(1.5,2,1.5)$ and $\psi^{\prime}=\operatorname{diag}(1.5,2,1.5)$. Figure 10 (a) shows the trajectories of errors, and it can be seen that the synchronization errors tend to zero; that is, the driving system (47) and the response system (48) achieve stable synchronization in $4 \mathrm{D}$. Figures 10(b) and 10(c) show the trajectories of the unknown parameter estimations; it can be seen that the estimations of unknown parameters can converge to some constants.

\section{Conclusions}

Based on Lyapunov stability theory and adaptive control method, the synchronization of chaotic systems with different dimensions is researched in this paper. Using two scaling matrices, an adaptive feedback hybrid controller and parameter adaptive laws are designed to realize the synchronization of chaotic systems with different dimensions. According to the characteristics of unknown parameters, a more simplified synchronization control scheme is designed. Finally, three examples of chaotic systems are used to demonstrate the effectiveness of the proposed method. Although a new controller is set up according to the characteristics of the system, it is still more complex. It is hoped that a more simple and convenient controller is designed for the synchronization of chaotic system.

\section{Data Availability}

No data were used to support this study.

\section{Conflicts of Interest}

The authors declare that they have no conflicts of interest.

\section{Acknowledgments}

This work was partly supported by the National Natural Science Foundation of China (Grant nos. 11901073 and 61876201).

\section{References}

[1] K. T. Alligood, T. D. Sauer, and Yorke, Chaos: An Introduction to Dynamical Systems, Springer, Berlin, Germany, 1997.
[2] L. M. Pecora and T. L. Carroll, "Synchronization in chaotic systems," Physical Review Letters, vol. 64, no. 8, pp. 821-824, 1990.

[3] V. Behrouz, A. P. Mohammad, and M. Saleh, "Finite-time chaos synchronization and its application in wireless sensor networks," Transactions of the Institute of Measurement and Control, vol. 40, no. 13, pp. 3788-3799, 2017.

[4] M. Saleh, V. Sundarapandian, S. Aceng et al., "A novel chaotic system with boomerang-shaped equilibrium, its circuit implementation and application to sound encryption," Iranian Journal of Science and Technology-Transactions of Electrical Engineering, vol. 43, no. 1, pp. 1-12, 2019.

[5] H. Li, Y. Wang, and Z. Zuo, "Chaos-based image encryption algorithm with orbit perturbation and dynamic state variable selection mechanisms," Optics and Lasers in Engineering, vol. 115, no. 40, pp. 197-207, 2019.

[6] R. S. T. Lee and S. T. Raymond, "Chaotic type-2 transientfuzzy deep neuro-oscillatory network (CT2TFDNN) for worldwide financial prediction," IEEE Transactions on Fuzzy Systems, vol. 28, no. 4, pp. 731-745, 2020.

[7] Q. Ye, Z. X. Jiang, and T. Chen, “Adaptive feedback control for synchronization of chaotic neural systems with parameter mismatches," Complexity, vol. 2018, Article ID 5431987, 8 pages, 2018.

[8] H. S. Sha, G. J. Wang, T. Hao et al., "Projection synchronization of a class of complex chaotic systems with both uncertainty and disturbance," Complexity, vol. 2020, Article ID 3742876, 12 pages, 2020.

[9] L. Ren, R. Guo, and U. E. Vincent, "Coexistence of synchronization and anti-synchronization in chaotic systems," Archives of Control Sciences, vol. 26, no. 1, pp. 69-79, 2016.

[10] M. Omid, M. Saleh, and K. Mohammad-Hassan, "Sliding mode disturbance observer control based on adaptive synchronization in a class of fractional-order chaotic systems," International Journal of Adaptive Control and Signal Processing, vol. 33, no. 3, pp. 462-474, 2018.

[11] M. Saleh and J. Ma, "Robust finite-time composite nonlinear feedback control for synchronization of uncertain chaotic systems with nonlinearity and time-delay," Chaos Solitons and Fractals, vol. 114, no. 30, pp. 46-54, 2018.

[12] H. Tirandaz, S. S. Aminabadi, and H. Tavakoli, "Chaos synchronization and parameter identification of a finance chaotic system with unknown parameters, a linear feedback controller," Alexandria Engineering Journal, vol. 57, no. 3, pp. 1519-1524, 2018.

[13] A. Stefanovska, H. Haken, P. V. E. Mcclintock, M. Hožič, F. Bajrović, and S. Ribarič, "Reversible transitions between synchronization states of the cardiorespiratory system," Physical Review Letters, vol. 85, no. 22, pp. 4831-4834, 2000.

[14] S. Q. Pang, Y. Feng, and Y. J. Liu, "Finite-time synchronization of chaotic systems with different dimension and secure communication," Mathematical Problems in Engineering, vol. 2016, Article ID 7693547, 14 pages, 2016.

[15] J. Gao and X. H. Zhang, "Synchronization of chaotic systems with different dimensions based on adaptive control," Computer Engineering and Science, vol. 35, no. 3, pp. 178-182, 2013, in Chinese.

[16] T. Hamed and S. Saeid, "Identical and non-identical synchronization of three scroll unified chaotic system (TSUCS) with unknown parameter using a modified function projective control method," Iranian Journal of Science and Technology-Transactions of Electrical Engineering, vol. 41, no. 4, pp. 319-334, 2017. 
[17] M. M. Al-Sawalha and M. S. M. Noorani, “Adaptive reducedorder anti-synchronization of chaotic systems with fully unknown parameters," Communications in Nonlinear Science and Numerical Simulation, vol. 15, no. 10, pp. 3022-3034, 2010.

[18] M. M. Al-Sawalha and M. S. M. Noorani, "Adaptive increasing-order synchronization and anti-synchronization of chaotic systems with uncertain parameters," Chinese Physics Letters, vol. 28, no. 11, pp. 2852-2857, 2011.

[19] R. S. Xue and L. W. Zuo, "Adaptive added-order anti-synchronization of chaotic systems with fully unknown parameters," Applied Mathematics and Computation, vol. 215, no. 5, pp. 1711-1717, 2009.

[20] A. Ouannas and M. M. Al-sawalha, "Synchronization between different dimensional chaotic systems using two scaling matrices," Optik, vol. 127, no. 2, pp. 959-963, 2016.

[21] A. Ouannas, M. M. Al-sawalha, and T. Ziar, "Fractional chaos synchronization schemes for different dimensional systems with non-identical fractional-orders via two scaling matrices," Optik, vol. 127, no. 20, pp. 8410-8418, 2016.

[22] A. Ouannas, S. Bendoukha, A. A. Khennaoui, G. Grassi, X. Wang, and V.-T. Pham, "Chaos synchronization of fractional-order discrete-time systems with different dimensions using two scaling matricesfferent dimensions using two scaling matrices," Open Physics, vol. 17, no. 1, pp. 942-949, 2019.

[23] X. Guo, G. Wen, Z. Peng, and Y. Zhang, "Global fixed-time synchronization of chaotic systems with different dimensions," Journal of the Franklin Institute, vol. 357, no. 2, pp. 1155-1173, 2020.

[24] H. K. Khalil, Nonlinear Systems, Prentice-Hall, Hoboken, NJ, USA, 3rd edition, 2002.

[25] G. L. Cai, Z. M. Tan, and W. H. Zhou, "Dynamic analysis and chaos control of a new chaotic system," Physical Letters, vol. 56, no. 11, pp. 6230-6237, 2007, in Chinese.

[26] O. E. Rössler, "An equation for continuous chaos," Physics Letters A, vol. 57, no. 5, pp. 397-398, 1976.

[27] X. Li, X. Z. Liu, G. R. Chen et al., "A new hyperchaotic Lorenztype system: generation, analysis, and implementation," International Journal of Circuit Theory and Applications, vol. 39, no. 8, pp. 865-879, 2011.

[28] R. Wang, J. Deng, and Z. Jing, "Chaos control in Duffing system," Chaos, Solitons \& Fractals, vol. 27, no. 1, pp. 249-257, 2006.

[29] D. S. Huang and H. Q. Ling, Theory and Method of the Nonlinear Economics, Sichuan University Press, Chengdu, China, 1993, in Chinese.

[30] Y. W. Chen, "Finite time synchronization of hyperchaotic Chen-Lee system," Journal of Zhangzhou Normal University, vol. 22, no. 3, pp. 18-22, 2009, in Chinese. 